TRANSACTIONS OF THE

AMERICAN MATHEMATICAL SOCIETY

Volume 359, Number 4, April 2007, Pages 1445-1470

S 0002-9947(06)04117-1

Article electronically published on October 17, 2006

\title{
THE ALGEBRAIC ENTROPY OF THE SPECIAL LINEAR CHARACTER AUTOMORPHISMS OF A FREE GROUP ON TWO GENERATORS
}

\author{
RICHARD J. BROWN
}

\begin{abstract}
In this note, we establish a connection between the dynamical degree, or algebraic entropy of a certain class of polynomial automorphisms of $\mathbb{R}^{3}$, and the maximum topological entropy of the action when restricted to compact invariant subvarieties. Indeed, when there is no cancellation of leading terms in the successive iterates of the polynomial automorphism, the two quantities are equal. In general, however, the algebraic entropy overestimates the topological entropy. These polynomial automorphisms arise as extensions of mapping class actions of a punctured torus $S$ on the relative $\mathrm{SU}(2)$-character varieties of $S$ embedded in $\mathbb{R}^{3}$. It is known that the topological entropy of these mapping class actions is maximized on the relative character variety comprised of reducible characters (those whose boundary holonomy is 2). Here we calculate the algebraic entropy of the induced polynomial automorphisms on the character varieties and show that it too solely depends on the topology of $S$.
\end{abstract}

\section{INTRODUCTION}

The topological entropy of an automorphism of a compact space is a measure of the orbit complexity of the points of the space under iteration of the automorphism. Positive entropy indicates complex dynamical behavior, and is a good indication that the dynamical structure of the system is interesting. For polynomial automorphisms of affine space, the dynamical degree was introduced by Bedford-Smillie [1] as a dynamical invariant for studying the complexities of the generalized Henon maps of $\mathbb{C}^{2}$. The logarithm of the dynamical degree is the algebraic entropy defined by Bellon-Viallet [2] for $p \in \operatorname{Poly} A u t\left(\mathbb{C}^{m}\right)$, the algebraic entropy $d_{p}$ is

$$
d_{p}=\log \lim _{n \rightarrow \infty}\left(\operatorname{deg} p^{n}\right)^{\frac{1}{n}} .
$$

For maps with complicated dynamics, the algebraic entropy is the (asymptotic) growth factor of the degree of an invertible polynomial map under iteration. In many cases, it is readily comparable to the topological entropy of the induced action on the compactification of affine space. For automorphisms of $\mathbb{C}$, the algebraic entropy equals the topological entropy of the action on the Julia set of the map. For $\mathbb{C}^{2}$ automorphisms, Smillie 23] proves the equality of topological and algebraic entropy when the map is extended to its one point compactification, answering a question by Friedland-Milnor [12]. Also, there is a current active study of the dynamics of polynomial maps of $\mathbb{C}^{n}$ and the corresponding rational maps of $\mathbb{C P}^{n}$, for

Received by the editors December 21, 2004.

2000 Mathematics Subject Classification. Primary 32M05.

(C)2006 American Mathematical Society Reverts to public domain 28 years from publication 
$n>2$ following Gromov [15] and Yomdin 24] (see for instance Guedj-Sibony [16], or Fornaess-Sibony [7]). However, calculating the degree of the forward iterates of a general polynomial automorphism of $\mathbb{C}^{n}, n>2$, is not so clear, due to possible issues like the cancellation of leading terms upon iteration, or degree-lowering. (In many cases of higher-dimensional maps, $\operatorname{deg} p^{n}<(\operatorname{deg} p)^{n}$ even without cancellation of terms. See below or Maegawa [19.) In this regard, the focus has been on the classification of polynomial maps as an aid in calculating the dynamical degree, as in Maegawa 20] and Fornaess-Wu [8] (compare also Bonifant-Fornaess [3]).

In this note, we establish a method to calculate the degree of the $n$th iterate of a special class of polynomial automorphisms of $\mathbb{C}^{3}$. We then use this to calculate the algebraic entropy. Specifically, let

$$
\kappa(x, y, z)=x^{2}+y^{2}+z^{2}-x y z-2
$$

be a cubic polynomial defined on $\mathbb{C}^{3}$. Denote by $\Sigma=A u t(\kappa)$ the group of polynomial automorphisms of $\mathbb{C}^{3}$ which leave invariant the fibers of $\kappa$. Evidently, $\Sigma$ is a representation of the group $P G L(2, \mathbb{Z}) \ltimes \Gamma$, where $\Gamma$ is the Klein 4-group of paired sign changes (see Goldman [13). For an element $\sigma \in \Sigma$, denote its projection in $P G L(2, \mathbb{Z})$ by $\sigma_{h}$ and its polynomial automorphism by $\varphi_{\sigma} \in \operatorname{Poly} A u t\left(\mathbb{C}^{3}\right)$. Also, for a matrix $A$, denote by $\operatorname{Spec}(A)$ its spectral radius. Here we show:

Theorem 1.1. For $\sigma \in \Sigma$, the algebraic entropy of $\varphi_{\sigma}$ is

$$
d_{\sigma}=\log \operatorname{Spec}\left(\sigma_{h}\right) .
$$

The automorphism group of $\kappa$ has been studied by Goldman-Neumann [14, Brown [5], Fried [1], and others in the context of the $S L(2, \mathbb{C})$-character variety of a torus with one boundary component $S$. In this context, the fundamental group $\pi_{1}(S)=F_{2}$, the free group on two generators. The $S L(2, \mathbb{C})$-character variety of $S$ is known to be all of $\mathbb{C}^{3}$, and the real points correspond to either $S L\left(\mathbb{R}^{2}\right)$ or $S U(2)$ characters of $F_{2}$ (Morgan-Shalen [21]). The set of all real characters is precisely $\mathbb{R}^{3}$, but is foliated by the subvarieties corresponding to characters whose evaluation on the boundary is fixed. The leaves of this foliation are the character varieties of $S$ relative to this fixed boundary condition, and are called the relative character varieties of $S$. In $F_{2}=\langle X, Y\rangle$, where the generators are chosen to coincide with a pair of simple closed curves that fill $S$, the commutator $X Y X^{-1} Y^{-1} \in F_{2}$ is homotopic to the boundary component. The polynomial $\kappa$ is the character of this commutator. The mapping class group of $S$, the group of isotopy classes of diffeomorphisms of $S$, necessarily leaves invariant the boundary. Here $M C G(S) \cong \operatorname{Out}\left(\pi_{1}(S)\right)=\operatorname{Out}\left(F_{2}\right)$ is isomorphic to $G L(2, \mathbb{Z})$ via the corresponding action on the integral first homology of $S$, and acts on characters as the group $P G L(2, \mathbb{Z})$. Thus $M C G(S)$ is commensurable with $A u t(\kappa)$ (Horowitz [18]), necessarily leaves invariant the boundary, and respects this foliation. For real characters, it is the $S U(2)$ characters which comprise the level-sets of $\kappa$ that contain compact components. Indeed, the $S U(2)$ character variety of $S$ is the intersection $\mathbb{R}^{3} \cap\left\{k^{-1}([-2,2])\right\}$. It is here that the topological entropy of elements of $A u t(\kappa)$ are studied.

Theorem 1.2. For $G \in A u t(\kappa)$, the algebraic entropy of $G$ is equal to the maximum of the topological entropies of $\left.G\right|_{\left\{\kappa^{-1}(r)\right\} \cap \mathbb{R}^{3}}$ for $\kappa^{-1}(r)$ a compact, invariant component of the $r$-level sets of $\kappa(x, y, z)$. Moreover, this maximum occurs on the compact real affine variety defined by $\left\{\kappa^{-1}(2)\right\} \cap[-2,2]^{3} \cap \mathbb{R}^{3}$. 
We prove these theorems by analyzing the growth of the degree of $\varphi_{\sigma}$ under iteration via its degree matrix $D_{\sigma}$ which we define in Section 2.3. Degree lowering is a common feature of these polynomial automorphisms of $\mathbb{C}^{3}$, and $D_{\sigma}$ correctly tracks the degree growth on iteration. Roughly speaking, a polynomial automorphism is constructive if there is no cancellation of its leading terms upon iteration. For an automorphism which is constructive, the growth of the degree of the iterates of $\varphi_{\sigma}$ are tracked via the column norm of the powers of $D_{\sigma}$, and are computed via a recursive relation built out of the characteristic equation of $D_{\sigma}$. In this case, $D_{\sigma^{n}}=D_{\sigma}^{n}$, and the characteristic equation of $D_{\sigma}$ is essentially that of $\sigma_{h}$ (up to a zero factor). As this computed invariant is also precisely the upper bound for the topological entropies of the action of $\sigma$ on the character varieties relative to the boundary of $S$, and this bound is achieved on the relative character variety consisting of the reducible characters (see Fried [11, for example), we relate the two explicitly.

In general, however, powers of the matrix $D_{\sigma}$ overestimate the degree of the iterates of $\varphi_{\sigma}$. This is due to cancellation of the leading terms upon iteration, thus further lowering the degree of $\varphi_{\sigma}^{n}$. An element $\sigma \in \operatorname{Out}\left(F_{2}\right)$ for which cancellation occurs is called nonconstructive. In this case, while the true degree of $\varphi_{\sigma}^{n}$ is still the column norm of $D_{\sigma^{n}}$, here $D_{\sigma^{n}} \neq D_{\sigma}^{n}$. However, the true growth of the degrees of the iterates of $\varphi_{\sigma}$ are still based on the topology of $\sigma$ (specifically the growth of the powers of $\sigma_{h}$ as manifested in the characteristic equation of $\sigma_{h}$ ). Here again, we use this information to compute the column norm of $D_{\sigma^{n}}$, and calculate the algebraic entropy of $\varphi_{\sigma}$.

The paper is organized as follows: In Section 2, we discuss some of the preliminaries. Here we detail the structure of the special linear character variety of a punctured torus, whose fundamental group is $F_{2}$. Also, we introduce the degree of the character of free group words, and describe the recursive growth of matrix entries under successive powers of the matrix. In Section 3, we define the degree matrix of a free group automorphism (technically, the polynomial automorphism induced by a free group automorphism, or mapping class), and discuss its properties. This allows us to classify which free group automorphisms are constructive. It also allows us to relate the entries of the degree matrix to those of the matrix which defines the linear transformation induced by $\sigma$ on the abelianization of $F_{2}$. In Section 4, we calculate explicitly the total degree of $\varphi_{\sigma^{n}}$ in terms of exponent counts of the images of the generators of $F_{2}$ under $\sigma^{n}$. The algebraic entropy of an automorphism is then computed via the recursive sequence formed from the characteristic equation. We then prove the theorems. Finally, we relegate some of the more technical aspects of our calculations to the Appendix. Here we prove the property of additivity of a free group automorphism, and establish criteria on whether a polynomial automorphism induced by a mapping class action is constructive or not.

\section{Preliminaries}

2.1. The $\mathrm{SL}(2, \mathbb{C})$-character variety of a surface. Let $S$ be the torus with one boundary component, such that $\pi_{1}(S)=F_{2}=\langle X, Y\rangle$, as shown in Figure 1.

Fricke 9 observed that the set of all characters of representations of $\pi_{1}(S)$ into $S L(2, \mathbb{C})$ is a closed affine set which naturally identifies with $\mathbb{C}^{3}$. Individually, the character of a free group word $W \in F_{2}=\pi_{1}(S)$ is a complex-valued function on 


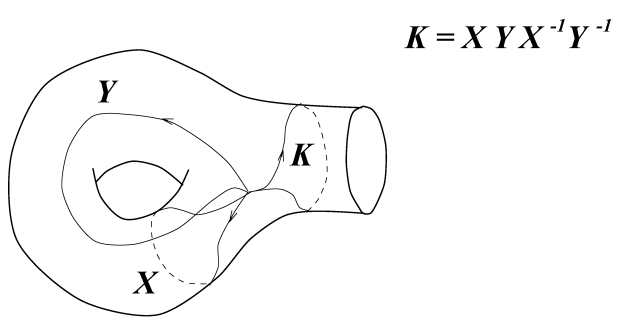

Figure 1. $\pi_{1}(S)=\langle X, Y\rangle=F_{2}$.

the set of all representations of $F_{2}$ into $S L(2, \mathbb{C})$,

$$
\operatorname{tr} W: \operatorname{Hom}\left(F_{2}, S L(2, \mathbb{C})\right) \rightarrow \mathbb{C}, \quad \operatorname{tr} W(\rho)=\operatorname{tr} \rho(W),
$$

where "tr" means the standard trace for special linear matrices. It was shown by Fricke and Klein [10] that for any cyclically reduced word $W \in F_{2}$, the special linear character of $W$ can be written as a polynomial with integer coefficients in the three characters

$$
x=\operatorname{tr} X, \quad y=\operatorname{tr} Y \quad z=\operatorname{tr} X Y .
$$

As (trace) coordinates, these three characters parameterize the character variety $\mathbb{C}^{3}\left(\mathbb{R}^{3}\right.$ when restricted to real-valued representations).

Homeomorphisms of $S$ induce isomorphisms of $\pi_{1}(S)$, and it is known that all isomorphisms of $\pi_{1}(S)$ are induced this way. Isomorphisms of $\pi_{1}(S)$ take characters to characters, and the inner automorphisms of $\pi_{1}(S)$ (those induced by homeomorphisms isotopic to the identity) act trivially on characters. Hence there is an action of the outer automorphism group $\operatorname{Out}\left(F_{2}\right)$, or equivalently the mapping class group of $S, M C G(S)$ on $\mathbb{C}^{3}$ and $\mathbb{R}^{3}$.

Homeomorphisms of $S$ necessarily take $\partial S$ to itself, and hence leave invariant the word $K \in \pi_{1}(S)$ homotopic to $\partial S$. Given the above presentation of $\pi_{1}(S)$, $K=X Y X^{-1} Y^{-1}$, and in the above trace coordinates,

$$
\operatorname{tr} K=\kappa(x, y, z)=-x y z+x^{2}+y^{2}+z^{2}-2 .
$$

By Horowitz [18], $\operatorname{Out}\left(\pi_{1}(S)\right)$ is commensurable with the action of the group Aut $(\kappa)$, so that the level sets of $\kappa$ are invariant under the action of $O u t\left(F_{2}\right)$.

Given $\sigma \in M C G(S)$, the corresponding linear action on integral first homology is given by the homomorphism

$$
h: M C G(S) \longrightarrow G L(2, \mathbb{Z}),
$$

which is an isomorphism by Nielsen [22]. Denote the total exponent count of the generator $X$ in a word $W \in F_{2}$ by $\epsilon_{X}(W)$. Then a rule for $h$ is

$$
h(\sigma)=\left(\begin{array}{cc}
\epsilon_{X}(\sigma(X)) & \epsilon_{X}(\sigma(Y)) \\
\epsilon_{Y}(\sigma(X)) & \epsilon_{Y}(\sigma(Y))
\end{array}\right) \in \operatorname{Aut}\left(H_{1}(S)\right)=G L(2, \mathbb{Z}) .
$$

Denote the image of $\sigma$ under $h$ by $\sigma_{h}$.

Considered as automorphism classes on $\pi_{1}(S), M C G(S)$ is generated by the two Dehn twist maps and an involution:

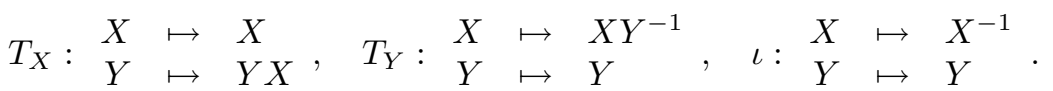


Note that the last generator above corresponds to a class of orientation-reversing homeomorphisms of $S$. Since these trace coordinates are actually functions on words in $F_{2}$, the action on characters is a pull-back of the action on words. The homomorphism

$$
\varphi: M C G(S) \longrightarrow \operatorname{Aut}(\kappa) \subset \operatorname{PolyAut}\left(\mathbb{C}^{3}\right)
$$

reverses the order of composition, and $\varphi(\sigma \circ \tau)=\varphi(\tau) \circ \varphi(\sigma)$. Moreover, the mapping class

$$
\begin{aligned}
\left(T_{Y} \circ T_{X} \circ T_{Y}\right)^{2}: X & \mapsto Y X^{-1} Y^{-1} \\
Y & \mapsto(Y X) Y^{-1}(Y X)^{-1}
\end{aligned}
$$

acts as the identity on characters (for any $\alpha \in \pi_{1}(S), \operatorname{tr} \alpha=\operatorname{tr} \alpha^{-1}$ ). This is due to the fact that $S$ is hyperelliptic (see, for instance, Goldman [13]). Hence the homomorphism $\varphi$ has a nontrivial kernel. It can be easily shown via the homology map $h$ that $\varphi$ factors through $P G L(2, \mathbb{Z})$. For $\sigma \in M C G(S)$, denote by $\varphi_{\sigma}$ its image as a polynomial automorphism of $\mathbb{C}^{3}$.

Using the above trace coordinates of $\mathbb{C}^{3}$, the mapping classes of (2.2) induce the polynomial automorphisms of $\mathbb{C}^{3}$ :

$$
\begin{aligned}
x & \mapsto x \\
\varphi_{T_{X}}: \begin{aligned}
x & \mapsto x y-z \\
y & \mapsto z \\
z & \mapsto z x-y
\end{aligned}, \varphi_{T_{Y}}: y & \mapsto y \\
z & \mapsto x
\end{aligned} \quad \begin{aligned}
x & \mapsto y \\
z & \mapsto y \\
z & \mapsto x y-z
\end{aligned}
$$

The compact components of the real level sets of $\kappa$ are comprised of the characters of $S U(2)$-representations. The $S U(2)$-character variety of $S$ in $\mathbb{R}^{3}$ is a set of concentric, topological 2-spheres parameterized by $\kappa \in[-2,2]$. (The origin is the level set $\kappa^{-1}(-2)$, while the outer sphere is the level set $\kappa^{-1}(2)$. See Brown [5].) Examples of a few of these level-set components are shown in the cutaway Figure 2. Fried calculated the topological entropy of mapping class actions on these level sets.

Theorem 2.1 (Fried [11]). For $\sigma \in A u t\left(F_{2}\right)$, the topological entropy $h_{T}\left(\left.\varphi_{\sigma}\right|_{\kappa^{-1}(r)}\right)$, for $r \in[-2,2]$, is maximized on the compact component of the level set $\kappa^{-1}(2)$, and here

$$
h_{T}\left(\left.\varphi_{\sigma}\right|_{\left\{\kappa^{-1}(2)\right\}}\right)=\log \operatorname{Spec}\left(\sigma_{h}\right) .
$$

2.2. The polynomial degree of a character. For $W \in F_{2}$, the special linear character $w$ is a polynomial with integer coefficients in the characters $x=\operatorname{tr} X$, $y=\operatorname{tr} Y$, and $z=\operatorname{tr} X Y$, and depends only on the character class of $W$. Hence, up to conjugacy and possibly inversion, replace $W$ with a member of its character class of the form

$$
W=X^{W_{1}} Y^{W_{2}} \cdots X^{W_{n-1}} Y^{W_{n}}
$$

where $W_{1}>0$ (compare Horowitz [18]). Under this "normalized" form for $W$, Brown 44 calculates the polynomial degree of $w$ directly via the syllable exponents $W_{i}$ of $W$. Denote by $\epsilon_{i}(w)$ the exponent of the $i$ th coordinate of the leading monomial of $w=\operatorname{tr} W$, for $i \in\{x, y, z\}$. (For a polynomial in $\mathbb{Z}[x, y, z]$ which arises as an $F_{2}$-character, there exists a unique monomial of highest total degree which is independent of the monomial ordering as long as it is graded.) Denote by $\operatorname{deg}(w)$ the total degree of this leading monomial. The following is proved in [4]. 


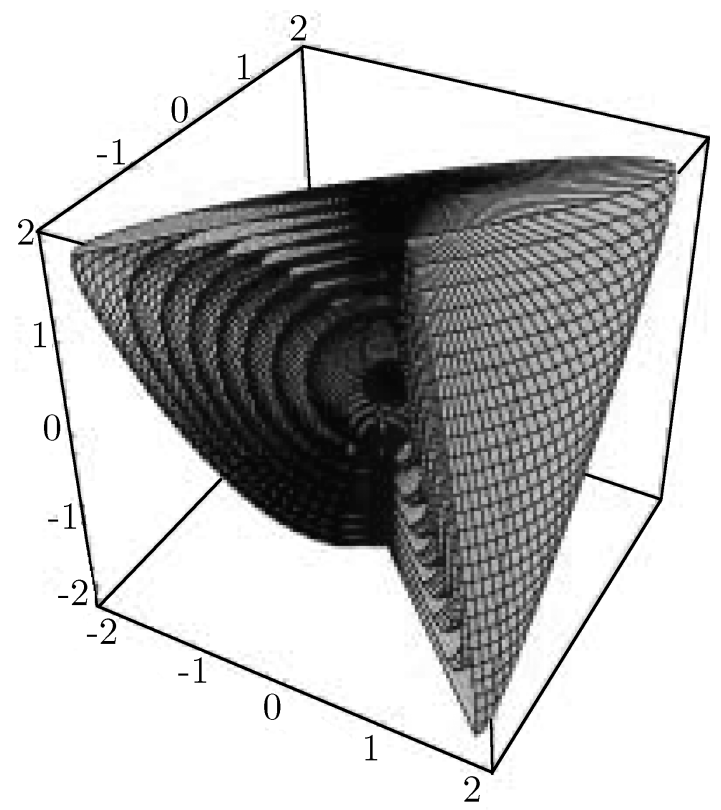

FIGURE 2. The SU(2)-character variety of $S$.

Lemma 2.2. $\operatorname{deg}(w)=\sum_{i=1}^{n}\left|W_{i}\right|-\sum_{i=1}^{n-1} r_{i}$, where

$$
r_{i}= \begin{cases}1 & \text { if }(-1)^{i-1} W_{i}>0 \text { and } W_{i} W_{i+1}>0, \\ 0 & \text { otherwise. }\end{cases}
$$

\section{Lemma 2.3.}

$$
\begin{aligned}
\epsilon_{x}(w) & =\sum_{i=1}^{\frac{n}{2}}\left|W_{2 i-1}\right|-\sum_{i=1}^{n-1} r_{i}, \\
\epsilon_{y}(w) & =\sum_{i=1}^{\frac{n}{2}}\left|W_{2 i}\right|-\sum_{i=1}^{n-1} r_{i}, \\
\epsilon_{z}(w) & =\sum_{i=1}^{n-1} r_{i} .
\end{aligned}
$$

2.3. Lucas sequences and matrix recursion. Let $A \in M(n, \mathbb{R})$ be an $n \times$ $n$ matrix with real coefficients. The Cayley-Hamilton form of its characteristic equation is

$$
A^{n}-c_{n-1} A^{n-1}+\cdots+(-1)^{n-2} c_{1} A+(-1)^{n-1} c_{0} I=0,
$$

where $c_{i}$ are fixed coefficients depending on $A$. Since this equation is simply a sum of matrices, the values of any sufficiently large power of $A$ is determined by the $n$th order recurrence equation

$$
\left(A^{n}\right)_{i j}=c_{n-1}\left(A^{n-1}\right)_{i j}-\cdots-(-1)^{n-2} c_{1} A_{i j}-(-1)^{n-1} c_{0} I_{i j} .
$$

More generally, for any $k \leq n$, the fixed $i j$ th entry of $A^{k}$ satisfies

$$
\left(A^{k}\right)_{i j}=c_{n-1}\left(A^{k-1}\right)_{i j}-\cdots-(-1)^{k-2} c_{1}\left(A^{k-(n-1)}\right)_{i j}-(-1)^{k-1} c_{0}\left(A^{k-n}\right)_{i j} .
$$


For $n=2$, the characteristic equation of $A \in M(2, \mathbb{R})$ takes the form

$$
A^{2}-c_{1} A+c_{0} I=0,
$$

where the coefficients satisfy $c_{1}=\operatorname{tr}(A)$, the trace of $A$, and $c_{0}=\operatorname{det}(A)$, the determinant. Hence the $i j$ th entry of the $n$th power $(n>1)$ of $A$ satisfies

$$
\left(A^{n}\right)_{i j}=(\operatorname{tr}(A)) \cdot\left(A^{n-1}\right)_{i j}-(\operatorname{det}(A)) \cdot\left(A^{n-2}\right)_{i j} .
$$

Call a second order recurrent sequence of the form

$$
x_{n}=a x_{n-1}+b x_{n-2}
$$

a Lucas sequence with coefficients $a$ and $b$, and denote the sequence $L(a, b)$. Thus any fixed position entry of $A \in M(2, \mathbb{R})$ under successive powers grows according to an $L(\operatorname{tr} A,-\operatorname{det} A)$ sequence.

Now consider the special case for $n=3$ with the restriction that the $\ell$ th row, for $\ell \in\{1,2,3\}$, is the zero vector. The singular matrix $A \in M(3, \mathbb{R})$ will be rank 2 provided the other two row vectors in $A$ are independent (and independent from the 0 -eigenvector). The characteristic equation for $A$ depends on the $2 \times 2$ cofactor $A_{\ell}$, formed by removing the $\ell$ th row and column from $A$. Indeed, the characteristic equation of $A$ is

$$
\lambda\left(\lambda^{2}-\left(\operatorname{tr} A_{\ell}\right) \lambda+\operatorname{det}\left(A_{\ell}\right)\right)=0 .
$$

The $n$th power of $A$ will still have the $\ell$ th row empty, and the entries of $A^{n}$ will satisfy the recursive relation

$$
\left(A^{n}\right)_{i j}=\left(\operatorname{tr} A_{\ell}\right)\left(A^{n-1}\right)_{i j}-\left(\operatorname{det} A_{\ell}\right)\left(A^{n-2}\right)_{i j} .
$$

Note immediately that (2.7) is Lucas $\left(\operatorname{tr} A_{\ell},-\operatorname{det} A_{\ell}\right)$.

Lemma 2.4. Let $A \in M(3, \mathbb{R})$ be such that the $\ell$ th row is the zero vector. Then the growth rate of any fixed position entry of $A$ under successive powers of $A$ is $L(a, b)$, where $a=\operatorname{tr}\left(A_{\ell}\right)$ and $b=-\operatorname{det}\left(A_{\ell}\right)$.

Let $A \in G L(2, \mathbb{Z})$. Then $\operatorname{det}(A)= \pm 1$, and the characteristic equation of $A$ is

$$
\lambda^{2}-a \lambda+b=0,
$$

where $a=\operatorname{tr} A$ and $\operatorname{det} A=b$. By the above discussion, this immediately implies that the entries of the positive powers of $A^{n}$ grow as $n$ grows according to an $L(a,-b)$ sequence. Let $s_{n}=\left(A^{n}\right)_{i j}, n \in \mathbb{Z}_{+} \cup\{0\}$, be the integer sequence corresponding to the fixed $i j$ position of the powers of $A$. Then under mild conditions, the absolute value of $\left\{s_{n}\right\}$ (at least the part of the sequence starting at $n=1$ ) is also a Lucas sequence.

Lemma 2.5. For $A \in S L(2, \mathbb{Z})$ hyperbolic, the sequence $\left\{s_{n}^{*}\right\}=\left\{\left|s_{n}\right|\right\}_{n=1}^{\infty}$ is $L(|a|,-1)$, and if $s_{0}>s_{1}$, then $s_{0}^{*}=-s_{0}$, otherwise $s_{0}^{*}=s_{0}$.

Proof. By the above discussion, $\left\{s_{n}\right\} \in L(a,-1)$. Since $A$ is special linear and hyperbolic with integer coefficients, $a \in \mathbb{Z}$ and $|a|>2$. Suppose $a$ is positive. Then $\left\{s_{n}\right\}$ is monotonic and increasing if and only if $s_{1} \geq s_{0}$ (we will leave this claim to the reader, noting that it is an easy consequence of the rule defining the sequence). If $\left\{s_{n}\right\}$ is increasing, then $s_{n}^{*}=\left|s_{n}\right|=s_{n}$, and there is nothing to prove. If $\left\{s_{n}\right\}$ is decreasing, then the entire sequence after the first term $s_{1}$ (which is not the 0th term $s_{0}$ ) is nonpositive, and $s_{n}^{*}=\left|s_{n}\right|=-s_{n}$. Recall that any multiple of 
a generalized Lucas sequence is also a generalized Lucas sequence with the same parameters. Choose $s_{0}^{*}=-s_{0}$, and we are done.

Now suppose $a$ is negative. Then $s_{n}$ is an alternating sequence. But for $A \in$ $S L(2, \mathbb{Z}),-A$ is also special linear, and satisfies the same characteristic equation. Hence for $s_{n}=\left(A^{n}\right)_{i j}$, where $\operatorname{tr} A<-2$, we have $\widehat{s}_{n}=\left((-A)^{n}\right)_{i j}$, where $\operatorname{tr}-A>2$. The sequence $\left\{\widehat{s}_{n}\right\}$ is monotonic, as in the case above, and $\widehat{s}_{n}= \pm\left|s_{n}\right|$, for $n \in \mathbb{Z}_{+}$ and $\widehat{s}_{0}=s_{0}$. Thus, we are back to the case where $a>2$ above. Choose $s_{n}^{*}=\left|\widehat{s}_{n}\right|=$ $\left|s_{n}\right|, n \in \mathbb{Z}_{+}$, and if $\widehat{s}_{n}$ is decreasing, choose $s_{0}^{*}=-s_{0}$, and $s_{0}^{*}=s_{0}$ otherwise.

Lemma 2.6. Let $A \in G L(2, \mathbb{Z})$ such that $\operatorname{tr} A \neq 0$ and $\operatorname{det} A=-1$. Then the sequence $\left\{s_{n}^{*}\right\}=\left\{\left|s_{n}\right|\right\}_{n=1}^{\infty}$ is $L(|a|, 1)$, and if $s_{0}>s_{1}$, then $s_{0}^{*}=-s_{0}$, otherwise $s_{0}^{*}=s_{0}$.

Proof. The proof is exactly the same as the one above, except that in this case, the sequence $\left\{s_{n}\right\}_{n \in \mathbb{Z}_{+}}$of either $A$ or $-A$ is monotonic as long as $\operatorname{tr} A \neq 0$. Hence it is not necessary that $|\operatorname{tr} A|>2$. The rest follows directly.

In the above cases of monotonically growing Lucas sequences, the growth is a sum of exponentials, whose bases are the (absolute values of the) roots of the characteristic equation. In these cases, the dominant root is the one of modulus greater than one. Ultimately, then, Lucas sequences of these types are asymptotically exponential, with growth factor the modulus of the dominant root.

Lemma 2.7. Let $\left\{s_{n}\right\}_{n \in \mathbb{Z}_{+}}$be an $L(a, 1)$ nonnegative integer sequence, where $s_{1}>$ $s_{0}$, and $a>2$. Then

where $\alpha$ is a root of

$$
\lim _{n \rightarrow \infty}\left(s_{n}\right)^{\frac{1}{n}}=\alpha>1,
$$

$$
\lambda^{2}-a \lambda+1=0 .
$$

Proof. It is a general fact that (2.9) is the characteristic equation of the Lucas sequence. The $n$th term of the sequence is then

$$
s_{n}=\frac{A \alpha^{n}-B \beta^{n}}{\alpha-\beta},
$$

where $\alpha$ and $\beta$ are the two distinct roots of (2.9) ( $a>2$ by supposition) and $A=s_{1}-s_{0} \beta$ and $B=s_{1}-s_{0} \alpha$. See Horadam [17]. Note here that since $\alpha \beta=1$ and $\alpha+\beta=a$, both $\alpha$ and $\beta=\frac{1}{\alpha}$ are positive. Choose $\alpha>1$ as the dominant root. Then

$$
s_{n}=\frac{A \alpha^{n}-B \frac{1}{\alpha^{n}}}{\alpha-\frac{1}{\alpha}}=\left[A \alpha^{n}-B \frac{1}{\alpha^{n}}\right] \cdot\left(\frac{\alpha}{\alpha^{2}-1}\right),
$$

so that

$$
\left(s_{n}\right)^{\frac{1}{n}}=\left[A \alpha^{n}-B \frac{1}{\alpha^{n}}\right]^{\frac{1}{n}} \cdot\left(\frac{\alpha}{\alpha^{2}-1}\right)^{\frac{1}{n}} .
$$

For $n$ very large, $\frac{1}{\alpha^{n}} \rightarrow 0$, and hence

$$
\left(s_{n}\right)^{\frac{1}{n}} \cong A^{\frac{1}{n}} \cdot\left(\alpha^{n}\right)^{\frac{1}{n}} \cdot\left(\frac{\alpha}{\alpha^{2}-1}\right)^{\frac{1}{n}},
$$

where both $A>0$ and $\frac{\alpha}{\alpha^{2}-1}>0$. Thus

$$
\lim _{n \rightarrow \infty}\left(s_{n}\right)^{\frac{1}{n}}=1 \cdot \alpha \cdot 1=\alpha .
$$


Remark 2.8. Note here that if $|a|=2$ above, then $\alpha=1$ and we cannot use the general $n$th term description of $s_{n}$. However, a sequence that satisfies

$$
s_{n+2}=2 s_{n+1}-s_{n}
$$

is arithmetic, and the terms will not approach exponential growth, as in the above case. Thus, we will again have

$$
\lim _{n \rightarrow \infty}\left(s_{n}\right)^{\frac{1}{n}}=\alpha=1 .
$$

Remark 2.9. Note also that if $|a|<2$ above, then the sequence is periodic and $\lim _{n \rightarrow \infty}\left(s_{n}\right)^{\frac{1}{n}}$ may not be defined.

Lemma 2.10. Let $\left\{s_{n}\right\}_{n \in \mathbb{Z}_{+}}$be $L(a, 1)$ nonnegative integer sequence, where $s_{1}>s_{0}$ and $a>0$. Then

where $\alpha$ is a root of

$$
\lim _{n \rightarrow \infty}\left(s_{n}\right)^{\frac{1}{n}}=\alpha>1,
$$

$$
\lambda^{2}-a \lambda-1=0 .
$$

Proof. Everything follows exactly from the lemma above, except that here it is not necessary for $a>2$, since the discriminant of (2.10) is $a^{2}+4$, which is always greater than 0 . This means there will always be two distinct real roots, with the positive one the dominant one.

\section{The DEGREE MATRIX OF A CHARACTER AUTOMORPHISM}

For any ring $R$ which includes the integers, let $p(x, y, z) \in R[x, y, z]$ be an $R$ polynomial in three indeterminates. In any graded monomial ordering on $R[x, y, z]$, denote by $\operatorname{lm}(p)$ the leading monomial of $p$. Call $\epsilon_{x}(p)$ the exponent of the coordinate $x$ in $\operatorname{lm}(p)$ (with similar definitions for $y$ and $z$ ). Then the degree of $p$ satisfies

$$
\operatorname{deg}(p)=\epsilon_{x}(p)+\epsilon_{y}(p)+\epsilon_{z}(p) .
$$

Generalize $p$ now to a polynomial map on $\mathbb{C}^{3}$ given by

$$
\begin{aligned}
& x \mapsto p_{x}(x, y, z) \\
& p: \mathbb{C}^{3} \rightarrow \mathbb{C}^{3}, \quad y \quad p_{y}(x, y, z) \\
& z \mapsto p_{z}(x, y, z) \text {. }
\end{aligned}
$$

One may record the degree and exponent count of the leading monomials of these coordinate functions via the $3 \times 3$ nonnegative integer matrix $D_{p}$, where

$$
D_{p}=\left(\begin{array}{ccc}
\epsilon_{x}\left(p_{x}\right) & \epsilon_{x}\left(p_{y}\right) & \epsilon_{x}\left(p_{z}\right) \\
\epsilon_{y}\left(p_{x}\right) & \epsilon_{y}\left(p_{y}\right) & \epsilon_{y}\left(p_{z}\right) \\
\epsilon_{z}\left(p_{x}\right) & \epsilon_{z}\left(p_{y}\right) & \epsilon_{z}\left(p_{z}\right)
\end{array}\right) .
$$

Call $D_{p}$ the degree matrix of $p$. The column norm (defined as the sum of the entries) of each column is the degree of each of the coordinate polynomials, and the column norm of the matrix $D_{p}$ - the maximum of the norms of each column - is the total degree of $p$. Recall that for $\sigma \in O u t\left(F_{2}\right)$, the corresponding polynomial automorphism of $\mathbb{C}^{3}$ is $\varphi_{\sigma}$, and the degree matrix of $\varphi_{\sigma}$ will be denoted $D_{\sigma}$, as mentioned in the Introduction, and

$$
D_{\sigma}=\left(\begin{array}{ccc}
\epsilon_{x}\left(\varphi_{\sigma}(x)\right) & \epsilon_{x}\left(\varphi_{\sigma}(y)\right) & \epsilon_{x}\left(\varphi_{\sigma}(z)\right) \\
\epsilon_{y}\left(\varphi_{\sigma}(x)\right) & \epsilon_{y}\left(\varphi_{\sigma}(y)\right) & \epsilon_{y}\left(\varphi_{\sigma}(z)\right) \\
\epsilon_{z}\left(\varphi_{\sigma}(x)\right) & \epsilon_{z}\left(\varphi_{\sigma}(y)\right) & \epsilon_{z}\left(\varphi_{\sigma}(z)\right)
\end{array}\right)
$$


In this section, we describe the structure of $D_{\sigma}$.

A priori, $D_{\sigma}$ may depend on the monomial ordering, since a different leading monomial would lead to different matrix entries. However, Brown 4 proves that the polynomial of a special linear character of a primitive word in $F_{2}$ has a unique monomial of maximum total degree. As the coordinate polynomials of $\varphi_{\sigma}$ are always the polynomials of characters of primitive words, under any graded monomial ordering, $D_{\sigma}$ will record the same entries, and hence is uniquely defined.

Let $\sigma \in \operatorname{Out}\left(F_{2}\right)$ generate a finite subgroup. Then so will $\varphi_{\sigma}$, and the dynamics of the action of $\varphi_{\sigma}$ on $\mathbb{C}^{3}$ will not be very interesting. The converse is also true. Indeed, if the action on characters is finite of order $n$, the $n$th iterate of the automorphism on free words will either take free words into conjugates of themselves (fixing the conjugacy class in $F_{2}$ ) or into other conjugacy classes of the same character. It was observed by Horowitz [18, however, that there are a finite number of conjugacy classes in every character class of $F_{2}$. Thus, as an outer automorphism, $\sigma$ will ultimately be finite. Hence, the focus of the rest of this discussion will be on elements $\sigma \in \operatorname{Out}\left(F_{2}\right)$ that generate infinite cyclic groups.

3.1. Topological description. Lemmas 2.2 and 2.3 relate the degree of the leading monomial of each of the coordinate polynomials of $\varphi_{\sigma}$ to the exponent sums in the image of the generators and their product under $\sigma$. This ties the entries of $D_{\sigma}$ to the topological description of $\sigma$. We make this precise via the following proposition. Recall that for any $W \in F_{2}, \epsilon_{X}(W)$ is the total exponent count of the letter $X$ in $W$, and $\epsilon_{x}(w)$ is the exponent of the coordinate $x$ in the leading monomial of the character $w=\operatorname{tr} W$, where $w \in \mathbb{Z}[x, y, z]$.

\section{Proposition 3.1.}

$$
\begin{aligned}
& \text { 1. If } \epsilon_{z}(w)=0, \text { then } \epsilon_{x}(w)=\left|\epsilon_{X}(W)\right| \text { and } \epsilon_{y}(w)=\left|\epsilon_{Y}(W)\right| \text {. } \\
& \text { 2. If } \epsilon_{x}(w)=0, \quad \text { then } \epsilon_{y}(w)=\left|\epsilon_{Y}(W)\right|-\left|\epsilon_{X}(W)\right| \\
& \text { 3. If } \epsilon_{y}(w)=0, \quad \text { then } \quad \epsilon_{z}(w)=\left|\epsilon_{X}(W)\right| . \\
& \text { and } \epsilon_{z}(w)=\left|\epsilon_{X}(W)\right|-\left|\epsilon_{Y}(W)\right|
\end{aligned}
$$

Proof. Let

$$
W=X^{W_{1}} Y^{W_{2}} \cdots X^{W_{n-1}} Y^{W_{n}}
$$

be normalized so that the first syllable has exponent $W_{1}>0$. To prove the first assertion, let $\epsilon_{z}(w)=0$. Then, by Lemma 2.3, $r_{i}=0$ for all $i \in\{1, \ldots, n\}$. It then follows that all of the exponents of the $X$ syllables in $W$ are positive, and all of the exponents of the $Y$ syllables are negative (otherwise a pairing $X Y$ or $Y^{-1} X^{-1}$ in $W$ would imply that for some $\left.i, r_{i}=1\right)$. Thus

$$
\epsilon_{x}(w)=\sum_{i=1}^{\frac{n}{2}}\left|W_{2 i-1}\right|=\left|\sum_{i=1}^{\frac{n}{2}} W_{2 i-1}\right|=\left|\epsilon_{X}(W)\right| .
$$

The same calculation for $Y$ yields $\epsilon_{y}(w)=\left|\epsilon_{Y}(W)\right|$.

Now let $\epsilon_{x}(w)=0$. Then again by Lemma 2.3

$$
\sum_{i=1}^{\frac{n}{2}}\left|W_{2 i-1}\right|=\sum_{i=1}^{n-1} r_{i}
$$


Recall that for each $i$ where $r_{i}=1$, we have $W_{i} W_{i+1}>0$ and $(-1)^{i-1} W_{i}>0$.

Suppose $r_{i}=1$, where $i$ is odd. Then $W_{i}>0$ is an $X$-exponent, and $W_{i+1}>0$. Since $i$ is odd, if $i \neq 1, r_{i-1}=0$, since $W_{i-1}<0$ and $W_{i-1} W_{i}>0$ cannot both be satisfied. Also, $r_{i+1}=0$, since $W_{i+1}>0$. Since the value of $r_{i}$ is 1 , this forces one of the letters in the syllable $X^{W_{i}}$ to pair with a $Y$. Since $\epsilon_{x}(w)=0$, this means that the only letter in the syllable $X^{W_{i}}$ is the single $X$, so that $W_{i}=1$.

If $i$ is even and $r_{i}=1$, then $W_{i}<0$ is a $Y$-exponent, and $W_{i+1}<0$. Again, this forces $r_{i-1}=0$ and $r_{i+1}=0$ (if this latter term exists), since $W_{i+1}<0$ in the latter case, and since $W_{i-1}>0$ and $W_{i-1} W_{i}>0$ cannot be simultaneously satisfied in the former. Again, since $r_{i}=1$, we must then have $W_{i+1}=-1$.

Hence every $X \in W$ is paired with a $Y$ of the form $X Y \subset W$, and every $X^{-1}$ is paired with a $Y^{-1}$ in the form $Y^{-1} X^{-1} \subset W$.

To see that both cannot occur within the same $W$ in the case where $\epsilon_{x}(w)=$ 0 , suppose that 2 consecutive $X$-exponents $W_{i}$ and $W_{i+2}$ satisfy $W_{i} W_{i+2}<0$. Referring to the form of $W$ given by

$$
W=\cdots Y^{W_{i-1}} X^{W_{i}} Y^{W_{i+1}} X^{W_{i+2}} Y^{W_{i+3}} \cdots
$$

(note that $W_{i-1}$ may be 0 here, if we are at the beginning of $W$ ), we may, by possibly inverting $W$, assume that $W_{i}>0$. Then $W_{i+2}<0$, forcing $r_{i-1}=0$ and $r_{i+2}=0$ immediately. By the above discussion, no matter what sign of the $Y$-exponent $W_{i+1}$ is, one of $r_{i}$ and $r_{i+1}$ must be 1 and the other 0 . Thus

$$
\left|W_{i}\right|+\left|W_{i+2}\right|>\sum_{j=i-1}^{i+2} r_{j}=1 .
$$

This contradiction shows that all of the $X$-exponents must be of the same sign. Also, if $\sum_{i=1}^{n-1} r_{i}>0$, all of the $Y$-exponents must also be of the same sign. Then it is straightforward to see that

$$
\begin{aligned}
\epsilon_{x}(w) & =0, \\
\epsilon_{y}(w) & =\left|\epsilon_{Y}(W)\right|-\left|\epsilon_{X}(W)\right|, \\
\epsilon_{z}(w) & =\left|\epsilon_{X}(W)\right| .
\end{aligned}
$$

A similar argument for the case $\epsilon_{y}(w)=0$ yields the following:

$$
\begin{aligned}
\epsilon_{x}(w) & =\left|\epsilon_{X}(W)\right|-\left|\epsilon_{Y}(W)\right|, \\
\epsilon_{y}(w) & =0, \\
\epsilon_{z}(w) & =\left|\epsilon_{Y}(W)\right| .
\end{aligned}
$$

This part of the construction we will leave for the reader.

Based on the information contained in Proposition 3.1 the entries of $D_{\sigma}$ are either the absolute values of entries of the homology matrix $\sigma_{h}$ or sums of them. Indeed, the character of $\sigma(I)$ for $I=X, Y$ is precisely $\varphi_{\sigma}(i)$. We will make this more precise after the following section.

3.2. Deficiency. A quick inspection of the generators of $O u t\left(F_{2}\right)$ in (2.4) reveals that the leading monomials of each of the coordinate polynomials together are functions of only two of the three coordinates. Any $\sigma \in \operatorname{Out}\left(F_{2}\right)$ is a composition of these generators and/or their inverses. If there is no cancellation of leading terms in the composition down to the point that the polynomial automorphism is linear, 
then the coordinate polynomials of a composition of these generators will also have this property. This is assured in the case that $\sigma$ is not a finite mapping class.

Lemma 3.2. If $\sigma \in \operatorname{Out}\left(F_{2}\right)$ generates an infinite cyclic subgroup, then there exists an $i \in\{x, y, z\}$, such that $\epsilon_{i}\left(\varphi_{\sigma}(j)\right)=0$, for all $j=x, y, z$.

Remark 3.3. Any $\varphi_{\sigma}$ is a word in the generators of (2.4) and/or their inverses. If $\varphi_{\sigma}$ is of length greater than 1 , then $\sigma=T_{W}^{\alpha} \circ \tau$, where $\tau \in \operatorname{Out}\left(F_{2}\right), W \in\{X, Y\}$, and $\alpha \in\{-1,1\}$. Suppose $W=X$ and $\alpha=1$. Since $\varphi_{\sigma}=\varphi_{T_{X} \circ \tau}=\varphi_{\tau} \circ \varphi_{T_{X}}$, we have

$$
\begin{aligned}
x & \mapsto x & \mapsto \tau_{x}(x, z, x z-y) \\
\varphi_{T_{X} \circ \tau}: y & \mapsto z & \mapsto \tau_{y}(x, z, x z-y) \\
z & \mapsto x z-y & \mapsto \tau_{z}(x, z, x z-y) .
\end{aligned}
$$

By inspection, we can see that $\epsilon_{y}\left(\varphi_{\sigma}(j)\right)=0$ for all $j=x, y, z$. In general, it is conceivable that $\tau$ would cancel the quadratic term produced by $T_{X}$, thus leaving a linear automorphism. However, for an infinite cyclic $\sigma$, the general form of its coordinate polynomials negate the chance for a cancellation of leading terms which could violate the lemma above (again, see [4]).

Proof of Lemma 3.2. It is clear that an automorphism of a group takes a basis to another basis. Hence, for $F_{2}=\langle X, Y\rangle$ and $\sigma \in \operatorname{Out}\left(F_{2}\right), \sigma(X)$ and $\sigma(Y)$ form a basis for $F_{2}$. By Cohen et al. [6], $\sigma(X)$ and $\sigma(Y)$ have a "normalized" form (compare also Brown [4]) for $p, q \geq 1$

$$
\begin{aligned}
& \sigma(X)=X^{n_{1}} Y^{m_{1}} \cdots X^{n_{q}} Y^{m_{q}}, \\
& \sigma(Y)=X^{\alpha_{1}} Y^{\beta_{1}} \cdots X^{\alpha_{p}} Y^{\beta_{p}},
\end{aligned}
$$

such that there exists an $r>0$ and a $\delta= \pm 1$, and either

1: $m_{1}=\cdots=m_{q}=\delta \beta_{1}=\cdots=\delta \beta_{p}=1$, and $\left\{n_{1}, \ldots, n_{q}, \delta \alpha_{1}, \ldots, \delta \alpha_{p}\right\}=\{r, r+1\}$,

or

2: $n_{1}=\cdots=n_{q}=\delta \alpha_{1}=\cdots=\delta \alpha_{p}=1$, and $\left\{m_{1}, \ldots, m_{q}, \delta \beta_{1}, \ldots, \delta \beta_{p}\right\}=\{r, r+1\}$,

or

3: one of the first two cases occurs with either $X$ or $Y$ replaced by their respective inverses $X^{-1}$ or $Y^{-1}$ throughout.

With this, one need only calculate the exponent of each coordinate via Lemma 2.3 for each of these cases. Indeed, in case 1, all of the exponents of $X$ and $Y$ are of the same sign in each word, and each $X$ is paired with a $Y$. The same is true for $\sigma(X Y)$, as it is simply a concatenation of $\sigma(X)$ and $\sigma(Y)$. (If the signs are different in the two words, then much cancelation will occur.) Hence $\epsilon_{x}\left(\varphi_{\sigma}(j)\right)=0$. In case $2, \epsilon_{y}\left(\varphi_{\sigma}(j)\right)=0$, and in case 3 , where one of the generators is replaced by its inverse, then there will be no places where any of the $r_{i}$ s will be nonzero, and $\epsilon_{z}\left(\varphi_{\sigma}(j)\right)=0$.

Corollary 3.4. For any infinite cyclic $\sigma \in \operatorname{Aut}\left(F_{2}\right)$, the degree matrix $D_{\sigma}$ contains a row which is the zero vector.

Definition 3.5. For $i \in\{x, y, z\}$, and $\sigma \in \operatorname{Out}\left(F_{2}\right)$, call $\varphi_{\sigma}$ (and hence $\sigma$ ) $i$ deficient, if for all $j=x, y, z, \epsilon_{i}\left(\varphi_{\sigma}(j)\right)=0$. 
Remark 3.6. A linear polynomial automorphism may have roots which are not linear. In (2.3),$\sigma^{2}=\left(T_{X} \circ T_{Y} \circ T_{X}\right)^{2}$ induces the identity automorphism $\varphi_{\sigma^{2}}$. Its square root is $\sigma=\left(T_{X} \circ T_{Y} \circ T_{X}\right)$, such that $\varphi_{\sigma}$ is the quadratic involution which is $z$-deficient:

$$
\varphi_{\sigma}:\left\{\begin{aligned}
x & \mapsto y \\
y & \mapsto x \\
z & \mapsto x y-z
\end{aligned}\right.
$$

Also note here that the deficiency of $\varphi_{\sigma}$ is only a descriptor of the leading monomials of the automorphism, and not a statement on the lack of a coordinate in a polynomial in general.

Proposition 3.1 may now be combined with this notion of deficiency to create a coefficient matrix which we will call the deficiency matrix: Let $\lambda_{i}$ be the $3 \times 2$ coefficient matrix for a $\varphi_{\sigma}$ which is $i$-deficient, where

$$
\lambda_{x}=\left(\begin{array}{rr}
0 & 0 \\
-1 & 1 \\
1 & 0
\end{array}\right), \quad \lambda_{y}=\left(\begin{array}{rr}
1 & -1 \\
0 & 0 \\
0 & 1
\end{array}\right), \quad \lambda_{z}=\left(\begin{array}{ll}
1 & 0 \\
0 & 1 \\
0 & 0
\end{array}\right)
$$

and

$$
R_{\sigma}=\left(\begin{array}{ccc}
\left|\epsilon_{X}(\sigma(X))\right| & \left|\epsilon_{X}(\sigma(Y))\right| & \left|\epsilon_{X}(\sigma(X Y))\right| \\
\left|\epsilon_{Y}(\sigma(X))\right| & \left|\epsilon_{Y}(\sigma(Y))\right| & \left|\epsilon_{Y}(\sigma(X Y))\right|
\end{array}\right) .
$$

Then for $\sigma i$-deficient, we can write

$$
D_{\sigma}=\lambda_{i} \cdot R_{\sigma}
$$

3.3. Additivity. The total exponent count of any word in $F_{2}$ is additive upon multiplication (composition) in the group. That is, for $W, V \in F_{2}$,

$$
\epsilon_{I}(W V)=\epsilon_{I}(W)+\epsilon_{I}(V)
$$

where $I \in\{X, Y\}$. In terms of $\sigma \in O u t\left(F_{2}\right)$,

$$
\epsilon_{I}(\sigma(X Y))=\epsilon_{I}(\sigma(X))+\epsilon_{I}(\sigma(Y))
$$

However, the exponent sum of the image of the product $X Y$ may be less than that of its factors (such is the case when $\delta=-1$ in the proof of Lemma 3.2 above). Due to cancellation, it will remain true that there is an additive relationship between the exponent sums of the images of the three words $X, Y$, and $X Y$, but for a particular $\sigma$, any of the three can be the dominant word (of largest reduced wordlength). In this section, we show that for any infinite cyclic $\sigma \in \operatorname{Out}\left(F_{2}\right)$, the leading monomial of $\varphi_{\sigma}$ will always be a product of the leading monomials of the other two coordinate functions. In terms of the degree matrix $D_{\sigma}$, this means that one column will always be the sum of the other two. Indeed, in Appendix A, we prove:

Proposition 3.7. Let $i$ be a Mod 3 index on the ordered coordinates $\{x, y, z\}$ and let $\sigma \in \operatorname{Out}\left(F_{2}\right)$ be infinite cyclic. Then there exists a value of $i \in\{x, y, z\}$, such that for all $j=x, y, z$,

$$
\epsilon_{j}\left(\varphi_{\sigma}(i)\right)=\epsilon_{j}\left(\varphi_{\sigma}(i+1)\right)+\epsilon_{j}\left(\varphi_{\sigma}(i+2)\right)
$$

Definition 3.8. For $i \in\{x, y, z\}$, and $\sigma \in O u t\left(F_{2}\right)$, call $\varphi_{\sigma}$-additive if the equation in Proposition 3.7 holds for $i$. 
In (3.2), the matrix $R_{\sigma}$ would display the additivity of $\sigma$ (equivalently $\varphi_{\sigma}$ ), in the sense that the $j$ th column of $R_{\sigma}$ is a term-by-term sum of the other two when $\sigma$ is $j$-additive. This property is passed through to $D_{\sigma}$.

3.4. Constructivity. Here, an infinite cyclic $\sigma \in \operatorname{Out}\left(F_{2}\right)$ is classified into one of two distinct types, based upon how the degree matrix $D_{\sigma}$ of the polynomial automorphism $\varphi_{\sigma}$ behaves under iteration of $\sigma$. This classification revolves around whether there is a cancellation of leading terms which reduces the degree of the future iterates of $\varphi_{\sigma}$.

The degree matrix records the degree of each coordinate polynomial in an automorphism via the column norm. The $n$th iterate of $\sigma \in \operatorname{Out}\left(F_{2}\right)$ induces a polynomial automorphism whose degree is the column norm of $D_{\sigma^{n}}$. It is tempting to assume that $D_{\sigma^{n}}=D_{\sigma}^{n}$, and the degree of the automorphism induced by $\sigma^{n}$ is simply the column norm of the $n$th power of $D_{\sigma}$. However, in general, this is not the case.

Intuitively, a polynomial automorphism is constructive if the degree of its iterates grows constructively. That is, if there are no cancellations of its leading terms upon iteration of the automorphism. Call $\sigma \in \operatorname{Out}\left(F_{2}\right)$ constructive if its induced polynomial automorphism $\varphi_{\sigma}$ is constructive.

An algebraic criterion for a polynomial automorphism to be constructive is the following, which we will use as a definition.

Lemma 3.9. $\sigma \in \operatorname{Out}\left(F_{2}\right)$ is constructive iff $D_{\sigma^{n}}=D_{\sigma}^{n}$.

Example 3.10 (of a nonconstructive $\sigma \in \operatorname{Out}\left(F_{2}\right)$ ). Let $\sigma \in \operatorname{Out}\left(F_{2}\right)$ be given by

$$
\begin{aligned}
X=T_{X}^{4} \circ T_{Y}: \quad & \mapsto X^{-3} Y^{-1} \\
Y & \mapsto Y X^{4} \\
X Y & \mapsto X^{-3} Y^{-1} Y X^{4}=X .
\end{aligned}
$$

Note that we include the image of $X Y \in \sigma$ for clarity. The induced automorphism $\varphi_{\sigma}$ is given by

$$
\begin{aligned}
x & \mapsto x^{2} z-x y-z \\
\varphi_{\sigma}: y & \mapsto x^{3} z-x^{2} y-2 x z+y \\
z & \mapsto x .
\end{aligned}
$$

Here the leading monomial of $\varphi_{\sigma}$ is the monomial $x^{3} z$ in the coordinate function $\sigma_{y}$. Note that the variable $y$ is absent from all of the leading monomials of the coordinate functions, consistent with Lemma 3.2, By easy calculation,

$$
D_{\sigma}=\left(\begin{array}{ccc}
2 & 3 & 1 \\
0 & 0 & 0 \\
1 & 1 & 0
\end{array}\right) \text { and } D_{\sigma}^{2}=\left(\begin{array}{ccc}
5 & 7 & 2 \\
0 & 0 & 0 \\
2 & 3 & 1
\end{array}\right) \text {. }
$$

However, the $x$-coordinate function of $\varphi_{\sigma^{2}}$ is

$$
\begin{aligned}
\varphi_{\sigma^{2}}(x) & =\varphi_{\sigma}\left(x^{2}-x y-z\right) \\
& =\left(x^{2}-x y-z\right)^{2} x-\left(x^{2} z-x y-z\right)\left(x^{3} z-x^{2} y-2 x z+y\right)-x \\
& =x^{5} z^{2}-2 x^{4} y z-\cdots-x^{5} z^{2}+2 x^{4} y z+\cdots-x \\
& =x^{3} z^{2}-2 x^{2} y z+x y^{2}-x z^{2}+y z-x .
\end{aligned}
$$

The column norm of the first column of $D_{\sigma}^{2}$ records the degree of the $x$-coordinate polynomial of $\varphi_{\sigma^{2}}$ as 7 , but neglects the fact that this leading term has been cancelled out by another term of the opposite coefficient. The leading term of the 
$x$-coordinate function of $\varphi_{\sigma^{2}}$ is actually $x^{3} z^{2}$, so that the first column of $D_{\sigma^{2}}$ is actually $\left(\begin{array}{lll}3 & 0 & 2\end{array}\right)^{T}$, and the true degree matrix of $\sigma^{2}$ is

$$
D_{\sigma^{2}}=\left(\begin{array}{ccc}
3 & 5 & 2 \\
0 & 0 & 0 \\
2 & 3 & 1
\end{array}\right) .
$$

Example 3.11 (of a constructive $\sigma \in \operatorname{Out}\left(F_{2}\right)$ ). Let

$$
\begin{aligned}
& \sigma=T_{Y}^{-1} \circ T_{X}: \quad Y \mapsto X Y \\
& Y Y \mapsto X X Y \\
& X Y Y^{2} X Y,
\end{aligned}
$$

so that

$$
\begin{aligned}
x & \mapsto z \\
\varphi_{\sigma}: y & \mapsto y z-x \\
z & \mapsto z(y z-x)-y=y z^{2}-x z-y .
\end{aligned}
$$

It is a straightforward calculation to say

$$
\begin{aligned}
x & \mapsto y z^{2}-x z-y \\
\varphi_{\sigma^{2}}: y & \mapsto(y z-x)\left(y z^{2}-x z-y\right)-z \\
z & \mapsto(y z-x)\left(y z^{2}-x z-y\right)^{2}-z\left(y z^{2}-x z-y\right)-(y z-x),
\end{aligned}
$$

which can be reworked to

$$
\begin{aligned}
& \varphi_{\sigma^{2}}(x)=z(y z-x)-y \\
& \varphi_{\sigma^{2}}(y)=z(y z-x)^{2}-y(y z-x)-z \\
& \varphi_{\sigma^{2}}(z)=z^{2}(y z-x)^{3}-2 y z(y z-x)^{2}+\left(y^{2}-z^{2}\right)(y z-x)+x .
\end{aligned}
$$

A quick calculation also yields

$$
D_{\sigma}=\left(\begin{array}{ccc}
0 & 0 & 0 \\
0 & 1 & 1 \\
1 & 1 & 2
\end{array}\right) \text { and } D_{\sigma}^{2}=\left(\begin{array}{ccc}
0 & 0 & 0 \\
1 & 2 & 3 \\
2 & 3 & 5
\end{array}\right) \text {. }
$$

Note that $D_{\sigma}^{2}=D_{\sigma^{2}}$ We will see later that some properties of $\sigma$ will imply that $D_{\sigma}^{n}=D_{\sigma^{n}}$ for all positive integers $n$.

Note that in Example 3.10, $\varphi_{\sigma}$ is both $y$-additive and $y$-deficient (both can be visually determined by the degree matrix $D_{\sigma}$ ). In contrast, in Example 3.11, $\varphi_{\sigma}$ is $z$-additive and $x$-deficient. This provides a clue as to the criteria which determine if a given automorphism is constructive or not.

Theorem 3.12. For $\sigma \in \operatorname{Out}\left(F_{2}\right)$ infinite cyclic, and $i, j \in\{x, y, z\}$, let $\varphi_{\sigma}$ be $i$-deficient and $j$-additive. Then $\sigma$ is constructive iff $i \neq j$.

We will prove Theorem 3.12 in AppendixB. It states that if the leading monomial of $\varphi_{\sigma}$ occurs in the $i$ th coordinate function, and if that same $i$ th coordinate does not appear in any of the leading monomials of the coordinate functions, then $\sigma$ is not constructive, and upon iteration of $\varphi_{\sigma}$, cancellations of leading terms will occur, thereby reducing the degree of the iterates. The converse also holds.

In the case of a constructive $\sigma \in \operatorname{Out}\left(F_{2}\right)$, there is a direct relationship between the characteristic equation of $\sigma_{h}$ and that of $D_{\sigma}$.

Theorem 3.13. Let $\sigma \in O u t\left(F_{2}\right)$ be constructive. Then the characteristic equation of $D_{\sigma}$ is

$$
\lambda\left[\lambda^{2}-\left|\operatorname{tr} \sigma_{h}\right| \lambda+\operatorname{det} \sigma_{h}\right]=0 .
$$


Proof. Since $\sigma$ is constructive, $\varphi_{\sigma}$ is $i$-deficient for some $i \in\{x, y, z\}$. Hence, the $i$ th row of $D_{\sigma}$ is the 0 -vector. The characteristic polynomial of $D_{\sigma}$ is

$$
\lambda\left[\lambda^{2}-\left(\operatorname{tr} A_{i}\right) \lambda+\left(\operatorname{det} A_{i}\right)\right]=0,
$$

where $A_{i}$ is the $i$ th cofactor of $D_{\sigma}$. For the moment, assume it is the middle, or $y$ th row, so that $\varphi_{\sigma}$ is $y$-deficient. Then

$$
\begin{aligned}
\operatorname{tr} A_{y} & =\epsilon_{x}\left(\varphi_{\sigma}(x)\right)+\epsilon_{z}\left(\varphi_{\sigma}(z)\right), \\
\operatorname{det} A_{y} & =\epsilon_{x}\left(\varphi_{\sigma}(x)\right) \cdot \epsilon_{z}\left(\varphi_{\sigma}(z)\right)-\epsilon_{z}\left(\varphi_{\sigma}(x)\right) \cdot \epsilon_{x}\left(\varphi_{\sigma}(z)\right) .
\end{aligned}
$$

The entries of $\sigma_{h}$ are the total exponent counts of the generators $X$ and $Y$ in $\sigma(X)$ and $\sigma(Y)$. Thus

$$
\begin{aligned}
\operatorname{tr} \sigma_{h} & =\epsilon_{X}(\sigma(X))+\epsilon_{Y}(\sigma(Y)) \\
\operatorname{det} \sigma_{h} & =\epsilon_{X}(\sigma(X)) \cdot \epsilon_{Y}(\sigma(Y))-\epsilon_{X}(\sigma(Y)) \cdot \epsilon_{Y}(\sigma(X)) .
\end{aligned}
$$

Thus the theorem is proved in the case that $\varphi_{\sigma}$ is $y$-deficient once we show that

$$
\left|\operatorname{tr} \sigma_{h}\right|=\operatorname{tr} A_{y} \text { and } \operatorname{det} \sigma_{h}=\operatorname{det} A_{y} .
$$

To this end, we show the first part of (3.4):

$$
\left|\operatorname{tr} \sigma_{h}\right|=\left|\epsilon_{X}(\sigma(X))+\epsilon_{Y}(\sigma(Y))\right| .
$$

Since $\sigma$ is constructive, by Theorem $3.12 \varphi_{\sigma}$ cannot be $y$-additive. Assume for the moment that $\varphi_{\sigma}$ is $z$-additive. Then, by the constructions of Appendix A, $\epsilon_{X}(\sigma(X))$ and $\epsilon_{Y}(\sigma(Y))$ are of the same sign. Then

$$
\begin{aligned}
\left|\operatorname{tr} \sigma_{h}\right| & =\left|\epsilon_{X}(\sigma(X))+\epsilon_{Y}(\sigma(Y))\right| \\
& =\left|\epsilon_{X}(\sigma(X))\right|+\left|\epsilon_{Y}(\sigma(Y))\right| \\
& =\epsilon_{x}\left(\varphi_{\sigma}(x)\right)+\epsilon_{z}\left(\varphi_{\sigma}(x)\right)+\epsilon_{z}\left(\varphi_{\sigma}(y)\right)
\end{aligned}
$$

by Proposition 3.1 Since $\varphi_{\sigma}$ is $z$-additive,

$$
\epsilon_{z}\left(\varphi_{\sigma}(z)\right)=\epsilon_{z}\left(\varphi_{\sigma}(x)\right)+\epsilon_{z}\left(\varphi_{\sigma}(y)\right),
$$

so that

$$
\begin{aligned}
\left|\operatorname{tr} \sigma_{h}\right| & =\epsilon_{x}\left(\varphi_{\sigma}(x)\right)+\epsilon_{z}\left(\varphi_{\sigma}(x)\right)+\epsilon_{z}\left(\varphi_{\sigma}(y)\right) \\
& =\epsilon_{x}\left(\varphi_{\sigma}(x)\right)+\epsilon_{z}\left(\varphi_{\sigma}(z)\right) \\
& =\operatorname{tr} A_{y} .
\end{aligned}
$$

In the other case, where we assume $\varphi_{\sigma}$ is $x$-additive, $\epsilon_{X}(\sigma(X))$ and $\epsilon_{Y}(\sigma(Y))$ are of different signs, and

$$
\begin{aligned}
\left|\operatorname{tr} \sigma_{h}\right| & =\left|\epsilon_{X}(\sigma(X))+\epsilon_{Y}(\sigma(Y))\right| \\
& =|| \epsilon_{X}(\sigma(X))|-| \epsilon_{Y}(\sigma(Y))|| \\
& =\left|\epsilon_{x}\left(\varphi_{\sigma}(x)\right)+\epsilon_{z}\left(\varphi_{\sigma}(x)\right)-\epsilon_{z}\left(\varphi_{\sigma}(y)\right)\right| .
\end{aligned}
$$

The $x$-additivity of $\varphi_{\sigma}$ implies that

$$
\epsilon_{z}\left(\varphi_{\sigma}(x)\right)=\epsilon_{z}\left(\varphi_{\sigma}(y)\right)+\epsilon_{z}\left(\varphi_{\sigma}(z)\right),
$$


and thus again

$$
\begin{aligned}
\left|\operatorname{tr} \sigma_{h}\right| & =\left|\epsilon_{x}\left(\varphi_{\sigma}(x)\right)+\epsilon_{z}\left(\varphi_{\sigma}(x)\right)-\epsilon_{z}\left(\varphi_{\sigma}(y)\right)\right| \\
& =\left|\epsilon_{x}\left(\varphi_{\sigma}(x)\right)+\epsilon_{z}\left(\varphi_{\sigma}(z)\right)\right| \\
& =\epsilon_{x}\left(\varphi_{\sigma}(x)\right)+\epsilon_{z}\left(\varphi_{\sigma}(z)\right) \\
& =\operatorname{tr} A_{y}
\end{aligned}
$$

As for the second part of (3.4),

$$
\begin{aligned}
\operatorname{det} \sigma_{h}= & \epsilon_{X}(\sigma(X)) \cdot \epsilon_{Y}(\sigma(Y))-\epsilon_{X}(\sigma(Y)) \cdot \epsilon_{Y}(\sigma(X)) \\
= & {\left[\epsilon_{x}\left(\varphi_{\sigma}(x)\right)+\epsilon_{z}\left(\varphi_{\sigma}(x)\right)\right] \cdot \epsilon_{z}\left(\varphi_{\sigma}(y)\right) } \\
& -\left[\epsilon_{x}\left(\varphi_{\sigma}(y)\right)+\epsilon_{z}\left(\varphi_{\sigma}(y)\right)\right] \cdot \epsilon_{z}\left(\varphi_{\sigma}(x)\right) \\
= & \epsilon_{x}\left(\varphi_{\sigma}(x)\right) \cdot \epsilon_{z}\left(\varphi_{\sigma}(y)\right)+\epsilon_{z}\left(\varphi_{\sigma}(x)\right) \cdot \epsilon_{z}\left(\varphi_{\sigma}(y)\right) \\
& -\epsilon_{x}\left(\varphi_{\sigma}(y)\right) \cdot \epsilon_{z}\left(\varphi_{\sigma}(x)\right)-\epsilon_{z}\left(\varphi_{\sigma}(y)\right) \cdot \epsilon_{z}\left(\varphi_{\sigma}(x)\right) \\
= & \epsilon_{x}\left(\varphi_{\sigma}(x)\right) \cdot \epsilon_{z}\left(\varphi_{\sigma}(y)\right)-\epsilon_{x}\left(\varphi_{\sigma}(y)\right) \cdot \epsilon_{z}\left(\varphi_{\sigma}(x)\right) .
\end{aligned}
$$

On the other hand,

$$
\begin{aligned}
\operatorname{det} A_{y}= & \epsilon_{x}\left(\varphi_{\sigma}(x)\right) \cdot \epsilon_{z}\left(\varphi_{\sigma}(z)\right)-\epsilon_{x}\left(\varphi_{\sigma}(z)\right) \cdot \epsilon_{z}\left(\varphi_{\sigma}(x)\right) \\
= & \epsilon_{x}\left(\varphi_{\sigma}(x)\right) \cdot\left[\epsilon_{z}\left(\varphi_{\sigma}(x)\right)+\epsilon_{z}\left(\varphi_{\sigma}(y)\right)\right] \\
& -\epsilon_{z}\left(\varphi_{\sigma}(x)\right) \cdot\left[\epsilon_{x}\left(\varphi_{\sigma}(x)\right)+\epsilon_{x}\left(\varphi_{\sigma}(y)\right)\right] \\
= & \epsilon_{x}\left(\varphi_{\sigma}(x)\right) \cdot \epsilon_{z}\left(\varphi_{\sigma}(y)\right)+\epsilon_{x}\left(\varphi_{\sigma}(x)\right) \cdot \epsilon_{z}\left(\varphi_{\sigma}(x)\right) \\
& -\epsilon_{z}\left(\varphi_{\sigma}(x)\right) \cdot \epsilon_{x}\left(\varphi_{\sigma}(x)\right)-\epsilon_{z}\left(\varphi_{\sigma}(x)\right) \cdot \epsilon_{x}\left(\varphi_{\sigma}(y)\right) \\
= & \epsilon_{x}\left(\varphi_{\sigma}(x)\right) \cdot \epsilon_{z}\left(\varphi_{\sigma}(y)\right)-\epsilon_{z}\left(\varphi_{\sigma}(x)\right) \cdot \epsilon_{x}\left(\varphi_{\sigma}(y)\right) \\
= & \operatorname{det} \sigma_{h} .
\end{aligned}
$$

The calculations that would show that the above holds in the case that $\varphi_{\sigma}$ is either $x$-deficient, or $z$-deficient are similar, and we leave them for the reader.

Corollary 3.14. For $\sigma$ constructive, $\operatorname{Spec}\left(\sigma_{h}\right)=\operatorname{Spec}\left(D_{\sigma}\right)$.

For $\sigma$ nonconstructive, the characteristic equation of $D_{\sigma}$ is still related to that of $\sigma_{h}$, but the relationship is not one that allows for easy calculation of $D_{\sigma^{n}}$ in terms of $D_{\sigma}$ and its powers.

\section{Algebraic entropy}

To prove the main theorems of this paper, we first calculate the total degree of an arbitrary iterate of the polynomial automorphism induced by a given $\sigma \in \operatorname{Out}\left(F_{2}\right)$ in terms of the first homology of $F_{2}$. Then we will show that these degrees grow according to a Lucas sequence, which is asymptotically exponential (actually a sum of exponentials, one of which has growth factor greater than 1). The entropy is then simply the base of this dominant exponential growth.

4.1. The degree of $\varphi_{\sigma}$. The degree of a polynomial automorphism $\varphi_{\sigma}$ may now be calculated directly in terms of $\sigma_{h}$. Herein, we prove:

Theorem 4.1. For $\sigma \in O u t\left(F_{2}\right)$ infinite cyclic, let $\varphi_{\sigma}$ be $i$-deficient and $j$-additive. Then

$$
\left\|D_{\sigma}\right\|= \begin{cases}\left|\epsilon_{Y}(\sigma(J))\right| & \text { if } i=x \\ \left|\epsilon_{X}(\sigma(J))\right| & \text { if } i=y \\ \left|\epsilon_{X}(\sigma(J))\right|+\left|\epsilon_{Y}(\sigma(J))\right| & \text { if } i=z\end{cases}
$$


Proof. Recall $i$-deficiency means $\epsilon_{i}\left(\varphi_{\sigma}(k)\right)=0$, for $k=x, y, z$. Also $j$-additivity means

$$
\left\|D_{\sigma}\right\|=\sum_{k=1}^{3} \epsilon_{k}\left(\varphi_{\sigma}(j)\right)
$$

With Proposition 3.1 above and for example, if $i=x$,

$$
\begin{aligned}
\left\|D_{\sigma}\right\| & =\sum_{k=1}^{3} \epsilon_{k}\left(\varphi_{\sigma}(j)\right) \\
& =\epsilon_{y}\left(\varphi_{\sigma}(j)\right)+\epsilon_{z}\left(\varphi_{\sigma}(j)\right) \\
& =\left|\epsilon_{Y}(\sigma(J))\right|-\left|\epsilon_{X}(\sigma(J))\right|+\left|\epsilon_{X}(\sigma(J))\right| \\
& =\left|\epsilon_{Y}(\sigma(J))\right| .
\end{aligned}
$$

The other two cases are similar.

Hence, iterating the automorphism $\sigma$ allows us to calculate the degree of $\varphi_{\sigma^{n}}=$ $\varphi_{\sigma}^{n}$, which is simply $\left\|D_{\sigma^{n}}\right\|$. Note here again that only in the case where $\sigma$ is constructive is it true that $\left\|D_{\sigma^{n}}\right\|=\left\|D_{\sigma}^{n}\right\|$. It is only $\left\|D_{\sigma^{n}}\right\|$ that we need. For example, the degree of $\varphi_{\sigma}^{15}$, where $\sigma$ is $x$-deficient and $z$-additive, is

$$
\left\|D_{\sigma^{15}}\right\|=\left|\epsilon_{Y}\left(\sigma^{15}(X Y)\right)\right| \text {. }
$$

4.2. Proof of main theorems. We are now in a position to prove the main theorems. To this end, we begin with Theorem 1.1, as Theorem 1.2 will then follow directly. Recall that $\operatorname{Aut}(\kappa)$ is the automorphism group of the polynomial $\kappa$ in (1.1) and $\operatorname{Aut}(\kappa) \cong P G L(2, \mathbb{Z}) \ltimes \Gamma=\Sigma$, where $\Gamma$ is the Klein 4-group of paired sign changes on the coordinates.

Theorem 1.1. For $\sigma \in \Sigma$, the algebraic entropy of $\varphi_{\sigma}$ is

$$
d_{\sigma}=\log \operatorname{Spec}\left(\sigma_{h}\right) .
$$

Proof. Note that we only need consider $\sigma$ as part of the subgroup $P G L(2, \mathbb{Z}$ ) (actually $G L(2, \mathbb{Z}) \cong M C G(S)$ ), as the pairwise sign-change automorphisms are not generated by mapping classes, and will affect neither the degree of the coordinate polynomial leading monomials nor any possible cancellations of leading terms upon iteration. Thus we limit our discussion to $\sigma$ being a mapping class.

By definition,

$$
d_{\sigma}=\log \left[\lim _{n \rightarrow \infty}\left(\operatorname{deg}\left(\varphi_{\sigma}^{n}\right)\right)^{\frac{1}{n}}\right]=\log \left[\lim _{n \rightarrow \infty}\left\|D_{\sigma^{n}}\right\|^{\frac{1}{n}}\right] .
$$

By Theorem 4.1. $\left\|D_{\sigma^{n}}\right\|$ is a linear combination of the absolute values of entries of $\sigma_{h}^{n}$. Let $\sigma$ be a finite order mapping class. Then $\varphi_{\sigma}$ is also. Thus $0<\left\|D_{\sigma^{n}}\right\| \leq M$, for all $n \in \mathbb{Z}_{+}$, where $M$ is the maximum of the degrees of any of the iterates of $\varphi_{\sigma}$. In this case, the limit on the right-hand side of (4.2) exists and equals 1 , so that the entropy vanishes. Note here that for $\sigma$ to be finite, then either $\sigma_{h} \in S L(2, \mathbb{Z})$ and not hyperbolic, or $\operatorname{det} \sigma_{h}=-1$ and $\operatorname{tr} \sigma_{h}=0$.

The entropy will also vanish in the case that the degrees of the iterates of $\varphi_{\sigma}$ grow arithmetically (as is the case when $\sigma$ is a parabolic element of $S L(2, \mathbb{Z}$ ), such as when it is one of the generating Dehn twists). Outside of these cases, $\sigma$ is infinite cyclic, and the entries of the sequence of forward powers of $\sigma_{h}$ grow as an $L\left(\operatorname{tr} \sigma_{h}\right.$, det $\left.\sigma_{h}\right)$ sequence. Also, by Lemmas 2.5 and 2.6. the absolute values of these entries are $L\left(\left|\operatorname{tr} \sigma_{h}\right|, \operatorname{det} \sigma_{h}\right)$ sequences. Hence $\left\|D_{\sigma^{n}}\right\|$ also grows according 
to the Lucas sequence of the same parameters as $n$ grows. Hence by Lemma 2.7 or Lemma 2.10.

$$
d_{\sigma}=\log \alpha,
$$

where $\alpha=\operatorname{Spec}\left(\sigma_{h}\right)$.

And by Theorems 1.1 and 2.1 above, the other main theorem of the paper now holds, namely:

Theorem 1.2. For $G \in A u t(\kappa)$, the algebraic entropy of $G$ is equal to the maximum of the topological entropies of $\left.G\right|_{\left\{\kappa^{-1}(r)\right\} \cap \mathbb{R}^{3}}$ for $\kappa^{-1}(r)$ a compact, invariant component of the $r$-level sets of $\kappa(x, y, z)$. Moreover, this maximum occurs on the compact real affine variety defined by $\left\{\kappa^{-1}(2)\right\} \cap[-2,2]^{3} \cap \mathbb{R}^{3}$.

\section{Appendix A. Proof of AdDitivity}

In this section, we prove the additivity condition of the columns of the degree matrix $D_{\sigma}$, for $\sigma \in \operatorname{Out}\left(F_{2}\right)$ infinite cyclic. Indeed, we prove Proposition 3.7; For $\sigma \in \operatorname{Out}\left(F_{2}\right)$ infinite cyclic, there exists a value of the Mod 3 index $i$ on the ordered coordinates $\{x, y, z\}$, such that for $j=x, y, z$,

$$
\epsilon_{j}\left(\varphi_{\sigma}(i)\right)=\epsilon_{j}\left(\varphi_{\sigma}(i+1)\right)+\epsilon_{j}\left(\varphi_{\sigma}(i+2)\right) .
$$

Recall that by definition, we would then call $\varphi_{\sigma} i$-additive.

We start with some facts about $\sigma$ and its relationship with the exponent counts of the leading terms of the coordinate function of $\varphi_{\sigma}$. Recall that for any $W \in F_{2}$, $\epsilon_{X}(W)$ is the total exponent count of the letter $X$ in $W$, and $\epsilon_{x}\left(\varphi_{\sigma}(w)\right)$ is the exponent of the coordinate $x$ in the leading monomial of the character $w=\operatorname{tr} W$, where $w \in \mathbb{Z}[x, y, z]$. A direct corollary of Proposition 3.1 (whose proof is evident within the proof of the proposition) is the following.

Corollary A.1. If $\epsilon_{x}(w)=0$ or $\epsilon_{y}(w)=0$, then

$$
\epsilon_{X}(W) \cdot \epsilon_{Y}(W) \geq 0 \text {. }
$$

If $\epsilon_{z}(w)=0$, then

$$
\epsilon_{X}(W) \cdot \epsilon_{Y}(W) \leq 0 .
$$

For any choice of nonfinite $\sigma \in \operatorname{Out}\left(F_{2}\right)$, one of the following four conditions must hold:

$$
\begin{aligned}
& \epsilon_{X}(\sigma(X)) \cdot \epsilon_{Y}(\sigma(Y))>0 \text { and } \varphi_{\sigma} \text { is either } x \text {-deficient or } y \text {-deficient, } \\
& \epsilon_{X}(\sigma(X)) \cdot \epsilon_{Y}(\sigma(Y))<0 \text { and } \varphi_{\sigma} \text { is } z \text {-deficient, } \\
& \epsilon_{X}(\sigma(X)) \cdot \epsilon_{Y}(\sigma(Y))<0 \text { and } \varphi_{\sigma} \text { is either } x \text {-deficient or } y \text {-deficient, } \\
& \epsilon_{X}(\sigma(X)) \cdot \epsilon_{Y}(\sigma(Y))>0 \text { and } \varphi_{\sigma} \text { is } z \text {-deficient. }
\end{aligned}
$$

Remark A.2. It is entirely possible that either $\epsilon_{X}(\sigma(X))=0$ or $\epsilon_{Y}(\sigma(Y))=0$. However, for $\sigma$ to generate an infinite cyclic group, they cannot both be zero. Moreover, any positive power of $\sigma$ greater than 1 will result in neither being zero. Hence we discount the case where $\epsilon_{X}(\sigma(X)) \cdot \epsilon_{Y}(\sigma(Y))=0$ by possibly passing to $\sigma^{2}$.

Lemma A.3. For $I=X, Y$, we have:

$$
\begin{aligned}
& \text { Inequalities (A.1) or A.2 hold iff } \epsilon_{I}(\sigma(X)) \cdot \epsilon_{I}(\sigma(Y)) \geq 0 \text {. } \\
& \text { Inequalities (A.3) or A.4 hold iff } \epsilon_{I}(\sigma(X)) \cdot \epsilon_{I}(\sigma(Y)) \leq 0 \text {. }
\end{aligned}
$$


Proof. We prove the first assertion, and leave the second to the reader. Suppose (A.1) holds. Then, by supposition and Corollary A.1, this is equivalent to the system

$$
\begin{aligned}
& \epsilon_{X}(\sigma(X)) \cdot \epsilon_{Y}(\sigma(Y))>0, \\
& \epsilon_{X}(\sigma(X)) \cdot \epsilon_{Y}(\sigma(X)) \geq 0, \\
& \epsilon_{X}(\sigma(Y)) \cdot \epsilon_{Y}(\sigma(Y)) \geq 0 .
\end{aligned}
$$

It is obvious by inspection that all six terms must be of the same sign (when non-zero). Hence this system implies the result that $\epsilon_{i}(\sigma(X)) \cdot \epsilon_{i}(\sigma(Y)) \geq 0$. Comparably, if A.2) holds, then the equivalent system is

$$
\begin{aligned}
& \epsilon_{X}(\sigma(X)) \cdot \epsilon_{Y}(\sigma(Y))<0, \\
& \epsilon_{X}(\sigma(X)) \cdot \epsilon_{Y}(\sigma(X)) \leq 0, \\
& \epsilon_{X}(\sigma(Y)) \cdot \epsilon_{Y}(\sigma(Y)) \leq 0 .
\end{aligned}
$$

By sign chasing through these inequalities, it turns out that the first terms of each inequality must be of the same sign $\left(\epsilon_{X}(\sigma(Y))\right.$ may be 0$)$, which is opposite to the signs of the other terms. Hence again by inspection, the result holds.

Conversely, if $\epsilon_{i}(\sigma(X)) \cdot \epsilon_{i}(\sigma(Y)) \geq 0$, then

$$
\begin{aligned}
& \epsilon_{X}(\sigma(X)) \cdot \epsilon_{X}(\sigma(Y)) \geq 0, \\
& \epsilon_{Y}(\sigma(X)) \cdot \epsilon_{Y}(\sigma(Y)) \geq 0 .
\end{aligned}
$$

Also, since for any $\sigma$, we have $\operatorname{det} \sigma_{H}=1$,

$$
\epsilon_{X}(\sigma(X)) \cdot \epsilon_{Y}(\sigma(Y))=1+\epsilon_{X}(\sigma(Y)) \cdot \epsilon_{Y}(\sigma(X))
$$

and both products here cannot be of opposite signs (although one may be 0 ). If $\epsilon_{X}(\sigma(Y)) \cdot \epsilon_{Y}(\sigma(X)) \geq 0$, then $\epsilon_{X}(\sigma(X)) \cdot \epsilon_{Y}(\sigma(Y))>0$. Then A.1 will hold as long as $\varphi_{\sigma}$ is not $z$-deficient. By Corollary A.1, for $\varphi_{\sigma}$ to be $z$-deficient,

$$
\epsilon_{X}(\sigma(i)) \cdot \epsilon_{Y}(\sigma(i)) \leq 0,
$$

for $i=X, Y$, and at least one must be nonzero. But by supposition and (A.5), all 4 terms of A.5 must be of the same sign. Hence (A.6) cannot be satisfied, and $\varphi_{\sigma}$ must be either $x$-deficient or $y$-deficient.

Suppose now that $\epsilon_{X}(\sigma(Y)) \cdot \epsilon_{Y}(\sigma(X))<0$, which immediately implies via (A.5) that $\epsilon_{X}(\sigma(X)) \cdot \epsilon_{Y}(\sigma(Y)) \leq 0$. (Due to Remark A.2. we will assume that this last inequality is strict.) If in this case $\varphi_{\sigma}$ were either $x$-deficient, or $y$-deficient, then by Corollary A.1, again we would get $\epsilon_{X}(\sigma(i)) \cdot \epsilon_{Y}(\sigma(i)) \geq 0$, with at least one of the inequalities strict. This would give us the consistent system

$$
\begin{aligned}
& \epsilon_{X}(\sigma(Y)) \cdot \epsilon_{Y}(\sigma(X))<0, \\
& \epsilon_{X}(\sigma(X)) \cdot \epsilon_{Y}(\sigma(Y)) \leq 0, \\
& \epsilon_{X}(\sigma(X)) \cdot \epsilon_{Y}(\sigma(X)) \geq 0, \\
& \epsilon_{X}(\sigma(Y)) \cdot \epsilon_{Y}(\sigma(Y)) \geq 0 .
\end{aligned}
$$

While this system is consistent, it is not with the added supposition $\epsilon_{X}(\sigma(X))$. $\epsilon_{X}(\sigma(Y)) \geq 0$. Hence, in this case, $\varphi_{\sigma}$ must be $z$-deficient, and then A.2 holds.

The proof in the other two cases is entirely symmetric and is omitted for brevity.

Lemma A.4. For $I=X, Y, \varphi_{\sigma}$ is $z$-additive iff $\epsilon_{I}(\sigma(X)) \cdot \epsilon_{I}(\sigma(Y)) \geq 0$. 
Proof. As in the previous lemma, we will prove the case when the inequality is strict, noting that additivity is preserved under iteration of an automorphism, but a zero exponent count will not be preserved.

Given the general additivity of exponent counts in group compositions

$$
\epsilon_{I}(\sigma(X Y))=\epsilon_{I}(\sigma(X))+\epsilon_{I}(\sigma(Y))
$$

the condition

$$
\epsilon_{I}(\sigma(X)) \cdot \epsilon_{I}(\sigma(Y))>0
$$

implies that both terms on the right-hand side of (A.7) are of the same sign. This is equivalent to

$$
\begin{aligned}
\left|\epsilon_{I}(\sigma(X Y))\right| & =\left|\epsilon_{I}(\sigma(X))+\epsilon_{I}(\sigma(Y))\right| \\
& =\left|\epsilon_{I}(\sigma(X))\right|+\left|\epsilon_{I}(\sigma(Y))\right| .
\end{aligned}
$$

By Proposition 3.1 if $\varphi_{\sigma}$ is $z$-deficient (so that $e_{z}\left(\varphi_{\sigma}(j)\right)=0$ for $j=x, y, z$ ), then this immediately implies the result, since for $J=X, Y, X Y$,

$$
\left|\epsilon_{X}(\sigma(J))\right|=\epsilon_{x}\left(\varphi_{\sigma}(j)\right) \text { and }\left|\epsilon_{Y}(\sigma(J))\right|=\epsilon_{y}\left(\varphi_{\sigma}(j)\right) \text {. }
$$

If instead, $\varphi_{\sigma}$ is $x$-deficient $\left(e_{x}\left(\varphi_{\sigma}(j)\right)=0\right)$, then choosing $I=X$ in (A.9), we get, via Proposition 3.1 .

$$
\epsilon_{z}\left(\varphi_{\sigma}(z)\right)=\epsilon_{z}\left(\varphi_{\sigma}(x)\right)+\epsilon_{z}\left(\varphi_{\sigma}(y)\right),
$$

and for $I=Y$,

$$
\epsilon_{y}\left(\varphi_{\sigma}(z)\right)+\epsilon_{z}\left(\varphi_{\sigma}(z)\right)=\epsilon_{y}\left(\varphi_{\sigma}(x)\right)+\epsilon_{z}\left(\varphi_{\sigma}(x)\right)+\epsilon_{y}\left(\varphi_{\sigma}(y)\right)+\epsilon_{z}\left(\varphi_{\sigma}(y)\right),
$$

which, coupled with the previous equation shows that

$$
\epsilon_{y}\left(\varphi_{\sigma}(z)\right)=\epsilon_{y}\left(\varphi_{\sigma}(x)\right)+\epsilon_{y}\left(\varphi_{\sigma}(y)\right) .
$$

The case where $e_{y}\left(\varphi_{\sigma}(w)\right)=0$ is entirely symmetric and is left to the reader.

There is a similar statement for the case where $\epsilon_{I}(\sigma(X)) \cdot \epsilon_{I}(\sigma(Y)) \leq 0$, and $x$ or $y$-additivity. To prove this, we will first need the following.

Lemma A.5. Let $A=\left(\begin{array}{cc}a_{11} & 1 \\ a_{21} & a_{22}\end{array}\right) \in G L(2, \mathbb{Z})$ generate an infinite cyclic group. Then

$$
\left|a_{11}\right| \geq\left|a_{12}\right| \text { iff }\left|a_{21}\right| \geq\left|a_{22}\right|,
$$

and at least one of the inequalities is strict.

Proof. For $A$ to generate an infinite cyclic group, at least one of the following is true:

$$
\begin{aligned}
& a_{12} a_{22} \neq 0 \\
& a_{11} a_{21} \neq 0
\end{aligned}
$$


Suppose (A.10) is true. Then, using $R$ to denote an as yet unchosen direction for the inequality, assume $\left|a_{11}\right| R\left|a_{12}\right|$. We have the following:

$$
\begin{aligned}
& \left|a_{11}\right| R\left|a_{12}\right| \text { iff }\left|a_{11}\right| \cdot\left|a_{22}\right| R\left|a_{12}\right| \cdot\left|a_{22}\right| \\
& \text { iff }\left|a_{11} \cdot a_{22}\right| R\left|a_{12}\right| \cdot\left|a_{22}\right| \\
& \text { iff }\left|1+a_{12} \cdot a_{21}\right| R\left|a_{12}\right| \cdot\left|a_{22}\right| \\
& \text { iff }\left|1+a_{12} \cdot a_{21}\right| \cdot \frac{1}{\left|a_{12}\right|} R\left|a_{22}\right| \\
& \text { iff } \quad\left|\frac{1+a_{12} \cdot a_{21}}{a_{12}}\right| R\left|a_{22}\right| \\
& \text { iff }\left|\frac{1}{a_{12}}+a_{21}\right| R\left|a_{22}\right| .
\end{aligned}
$$

Now assume that $a_{12} \cdot a_{21} \geq 0$ (that is, they have the same sign if $a_{21} \neq 0$ ). Then

$$
\left|a_{21}\right|<\left|\frac{1}{a_{12}}+a_{21}\right| \text {. }
$$

In this case, choose $R$ to be $\leq$, and we get

$$
\left|a_{11}\right| \leq\left|a_{12}\right| \text { iff }\left|a_{21}\right|<\left|\frac{1}{a_{12}}+a_{21}\right| \leq\left|a_{22}\right|,
$$

which is equivalent to the desired result.

If, on the other hand, $a_{12} \cdot a_{21}<0$, so that they have different signs,

$$
\left|a_{21}\right|>\left|\frac{1}{a_{12}}+a_{21}\right|=|| \frac{1}{a_{12}}|-| a_{22}|| .
$$

Since $0<\left|\frac{1}{a_{12}}\right| \leq 1$ and the entries of $A$ are integers, we can choose $R$ to be $\geq$, and we get

$$
\left|a_{11}\right| \geq\left|a_{12}\right| \text { iff }\left|a_{21}\right|>\left|\frac{1}{a_{12}}+a_{21}\right| \geq\left|a_{22}\right|
$$

as desired.

If (A.11) is true, then an entirely symmetric argument reveals

$$
\left|a_{11}\right| R\left|a_{12}\right| \text { iff }\left|a_{21}\right| R\left|\frac{1}{a_{22}}+a_{11}\right| R\left|a_{22}\right|,
$$

where again $R$ is chosen with respect to the signs of $a_{11} \neq 0$ and $a_{22}$ :

$$
\begin{aligned}
& \text { If } \quad a_{11} \cdot a_{22}>0, \quad \text { then choose } R=\leq, \\
& \text { If } \quad a_{11} \cdot a_{22} \leq 0, \quad \text { then choose } R=\geq .
\end{aligned}
$$

In the context of this discussion, Lemma A.5 states that for any nonfinite order element of $\sigma \in \operatorname{Out}\left(F_{2}\right)$, its matrix $\sigma_{h}$ satisfies

$$
\left|\epsilon_{X}(\sigma(X))\right| \geq\left|\epsilon_{X}(\sigma(Y))\right| \text { iff }\left|\epsilon_{Y}(\sigma(X))\right| \geq\left|\epsilon_{Y}(\sigma(Y))\right| .
$$

Hence one column always dominates the other.

Lemma A.6. For $I=X, Y, \varphi_{\sigma}$ is either $x$-additive or $y$-additive iff $\epsilon_{I}(\sigma(X))$. $\epsilon_{I}(\sigma(Y)) \leq 0$. 
Proof. As in the previous lemma, given (A.7) and (A.8), note that the terms on the right-hand side are of opposite signs now by supposition. Hence

$$
\left|\epsilon_{I}(\sigma(X Y))\right|=|| \epsilon_{I}(\sigma(X))|-| \epsilon_{I}(\sigma(Y))||,
$$

and by Lemma A.5, one of the two terms on the right-hand side will dominate for both choices of $I=X, Y$.

Again, by Proposition 3.1, if $\varphi_{\sigma}$ is $z$-deficient, so that $e_{z}\left(\varphi_{\sigma}(j)\right)=0, j=x, y, z$, then

$$
\epsilon_{j}\left(\varphi_{\sigma}(z)\right)=\left|\epsilon_{j}\left(\varphi_{\sigma}(x)\right)-\epsilon_{j}\left(\varphi_{\sigma}(y)\right)\right| \text { for } j=x, y .
$$

This converts readily to one of

$$
\epsilon_{j}\left(\varphi_{\sigma}(z)\right)+\epsilon_{j}\left(\varphi_{\sigma}(y)\right)=\epsilon_{j}\left(\varphi_{\sigma}(x)\right) \quad \text { or } \quad \epsilon_{j}\left(\varphi_{\sigma}(z)\right)+\epsilon_{j}\left(\varphi_{\sigma}(x)\right)=\epsilon_{j}\left(\varphi_{\sigma}(y)\right),
$$

depending on which column of $\sigma_{h}$ dominates (as in (A.12)). But these are the definitions of $x$ and $y$-additivity, respectively.

If $\varphi_{\sigma}$ is $x$-deficient $\left(e_{x}\left(\varphi_{\sigma}(j)\right)=0\right)$, then choosing $I=X$, we get the same basic result:

$$
\epsilon_{z}\left(\varphi_{\sigma}(z)\right)=\left|\epsilon_{z}\left(\varphi_{\sigma}(x)\right)-\epsilon_{z}\left(\varphi_{\sigma}(y)\right)\right|,
$$

and one of the following two equations holds:

$$
\epsilon_{z}\left(\varphi_{\sigma}(z)\right)+\epsilon_{z}\left(\varphi_{\sigma}(y)\right)=\epsilon_{z}\left(\varphi_{\sigma}(x)\right) \text { or } \epsilon_{z}\left(\varphi_{\sigma}(z)\right)+\epsilon_{z}\left(\varphi_{\sigma}(x)\right)=\epsilon_{z}\left(\varphi_{\sigma}(y)\right) .
$$

Staying in this case, and choosing $I=Y$, we get

$$
\epsilon_{y}\left(\varphi_{\sigma}(z)\right)+\epsilon_{z}\left(\varphi_{\sigma}(z)\right)=\left|\epsilon_{y}\left(\varphi_{\sigma}(x)\right)+\epsilon_{z}\left(\varphi_{\sigma}(x)\right)-\left(\epsilon_{y}\left(\varphi_{\sigma}(y)\right)+\epsilon_{z}\left(\varphi_{\sigma}(y)\right)\right)\right| .
$$

Then, if the first column of $\sigma_{h}$ dominates (the $X$ counts are bigger than the $Y$ counts), this reduces to

$$
\epsilon_{y}\left(\varphi_{\sigma}(z)\right)+\epsilon_{z}\left(\varphi_{\sigma}(z)\right)+\epsilon_{y}\left(\varphi_{\sigma}(y)\right)+\epsilon_{z}\left(\varphi_{\sigma}(y)\right)=\epsilon_{y}\left(\varphi_{\sigma}(x)\right)+\epsilon_{z}\left(\varphi_{\sigma}(x)\right),
$$

which, when coupled with the valid part of (A.13), reduces to

$$
\epsilon_{y}\left(\varphi_{\sigma}(z)\right)+\epsilon_{y}\left(\varphi_{\sigma}(y)\right)=\epsilon_{y}\left(\varphi_{\sigma}(x)\right) .
$$

The case where the second column of $\sigma_{h}$ is bigger (the senses are reversed in (A.12) is proved in exactly the same way, as is the more general case where $\varphi_{\sigma}$ is $y$-deficient $\left(e_{y}\left(\varphi_{\sigma}(j)\right)=0\right)$. Again, we omit these parts of the proof to avoid redundancy.

Proof of Proposition 3.7. This proof is established upon recognition that the suppositions of Lemmas A.4 and A.6 are exhaustive. It may happen that for $I \in\{X, Y\}$,

$$
\epsilon_{I}(\sigma(X)) \cdot \epsilon_{I}(\sigma(Y))=0
$$

However, since $\sigma$ is assumed infinite cyclic, the other choice for $I$ must be a strict inequality. Thus additivity is achieved for all nonfinite $\sigma$, and Proposition 3.7 is proved. 


\section{Appendix B. Proof of constructivity}

In this section, we prove Theorem 3.12, which classifies which free group automorphisms $\sigma \in \operatorname{Out}\left(F_{2}\right)$ induce polynomial automorphisms $\varphi_{\sigma}$ for which there is no cancellation of leading terms upon iteration. While the cancellation of leading terms in the iteration of a polynomial automorphism does not ultimately change the algebraic entropy of $\varphi_{\sigma}$, it does affect the total degree of each iterate.

Proof of Theorem 3.12. By (3.2) above, we can write a klth element of the degree matrix $D_{\sigma^{n}}$ as

$$
\left(D_{\sigma^{n}}\right)_{k \ell}=\operatorname{row}_{k}\left(\lambda_{i}\right) \cdot \operatorname{col}_{\ell}\left(R_{\sigma}\right),
$$

where we assume that $\sigma$ and hence $\sigma^{n}$ are $i$-deficient. Expand this in terms of the entries of each of the matrices on the right-hand side:

$$
\begin{aligned}
\left(D_{\sigma^{n}}\right)_{k \ell}= & \left(\lambda_{i}\right)_{k 1}\left|\epsilon_{X}\left(\sigma^{n}(L)\right)\right|+\left(\lambda_{i}\right)_{k 2}\left|\epsilon_{Y}\left(\sigma^{n}(L)\right)\right| \\
= & \left(\lambda_{i}\right)_{k 1}\left|\epsilon_{X}\left(\sigma^{n-1}(X)\right) \cdot \epsilon_{X}(\sigma(L))+\epsilon_{X}\left(\sigma^{n-1}(Y)\right) \cdot \epsilon_{Y}(\sigma(L))\right| \\
& +\left(\lambda_{i}\right)_{k 2}\left|\epsilon_{Y}\left(\sigma^{n-1}(X)\right) \cdot \epsilon_{X}(\sigma(L))+\epsilon_{Y}\left(\sigma^{n-1}(Y)\right) \cdot \epsilon_{Y}(\sigma(L))\right|,
\end{aligned}
$$

where again $\ell=1,2,3$ respectively represents $x, y, z$, and respectively is the character of $L=X, Y, X Y$, and, on the level of homology, $\sigma_{h}^{n}=\sigma_{h}^{n-1} \cdot \sigma_{h}$.

For $\sigma$ constructive, $D_{\sigma^{n}}=D_{\sigma}^{n}$ is equivalent to $D_{\sigma^{n}}=D_{\sigma^{n-1}} \cdot D_{\sigma}$ (the proof of which we will leave for the reader). Here

$$
\begin{aligned}
\operatorname{row}_{k}\left(D_{\sigma^{n-1}}\right) \cdot \operatorname{col}_{\ell}\left(D_{\sigma}\right)= & \sum_{m=1}^{3}\left(D_{\sigma^{n-1}}\right)_{k m} \cdot\left(D_{\sigma}\right)_{m \ell} \\
= & \sum_{m=1}^{3}\left[\left(\lambda_{i}\right)_{k 1}\left|\epsilon_{X}\left(\sigma^{n-1}(M)\right)\right|+\left(\lambda_{i}\right)_{k 2}\left|\epsilon_{Y}\left(\sigma^{n-1}(M)\right)\right|\right] \\
& \cdot\left[\left(\lambda_{i}\right)_{m 1}\left|\epsilon_{X}(\sigma(L))\right|+\left(\lambda_{i}\right)_{m 2}\left|\epsilon_{Y}(\sigma(L))\right|\right] .
\end{aligned}
$$

The basis for this proof is to show that

$$
\left(D_{\sigma^{n}}\right)_{k \ell}=\operatorname{row}_{k}\left(D_{\sigma^{n-1}}\right) \cdot \operatorname{col}_{\ell}\left(D_{\sigma}\right)
$$

holds precisely when and only when $i \neq j$, for $\sigma i$-deficient and $j$-additive.

To proceed, let $\sigma$ be $z$-deficient. Then

$$
\begin{aligned}
\operatorname{row}_{k}\left(D_{\sigma^{n-1}}\right) \cdot \operatorname{col}_{\ell}\left(D_{\sigma}\right)= & {\left[\left(\lambda_{z}\right)_{k 1}\left|\epsilon_{X}\left(\sigma^{n-1}(X)\right)\right|+\left(\lambda_{z}\right)_{k 2}\left|\epsilon_{Y}\left(\sigma^{n-1}(X)\right)\right|\right] \cdot\left|\epsilon_{X}(\sigma(L))\right| } \\
& +\left[\left(\lambda_{z}\right)_{k 1}\left|\epsilon_{X}\left(\sigma^{n-1}(Y)\right)\right|+\left(\lambda_{z}\right)_{k 2}\left|\epsilon_{Y}\left(\sigma^{n-1}(Y)\right)\right|\right] \cdot\left|\epsilon_{Y}(\sigma(L))\right| \\
= & \left(\lambda_{z}\right)_{k 1}\left[\left|\epsilon_{X}\left(\sigma^{n-1}(X)\right) \cdot \epsilon_{X}(\sigma(L))\right|+\left|\epsilon_{X}\left(\sigma^{n-1}(Y)\right) \cdot \epsilon_{Y}(\sigma(L))\right|\right] \\
+ & \left(\lambda_{z}\right)_{k 2}\left[\left|\epsilon_{Y}\left(\sigma^{n-1}(X)\right) \cdot \epsilon_{X}(\sigma(L))\right|+\left|\epsilon_{Y}\left(\sigma^{n-1}(Y)\right) \cdot \epsilon_{Y}(\sigma(L))\right|\right] .
\end{aligned}
$$

If $\sigma$ is $z$-deficient, then by Corollary A.1 $\epsilon_{X}(\sigma(L)) \cdot \epsilon_{Y}(\sigma(L)) \leq 0$ for all $n \geq 1$ (and strict inequality for $n>1$ ). Also, if $\sigma$ is $x$-additive or $y$-additive, then by LemmaA.6 $\epsilon_{L}(\sigma(X)) \cdot \epsilon_{L}(\sigma(Y)) \leq 0$. Therefore, by checking signs of the individual terms above, within each pair of square brackets, each of the products are of the 
same sign (or 0). Thus,

$$
\begin{aligned}
\operatorname{row}_{k}\left(D_{\sigma^{n-1}}\right) \cdot \operatorname{col}_{\ell}\left(D_{\sigma}\right)= & \left(\lambda_{i}\right)_{k 1}\left|\epsilon_{X}\left(\sigma^{n-1}(X)\right) \cdot \epsilon_{X}(\sigma(L))+\epsilon_{X}\left(\sigma^{n-1}(Y)\right) \cdot \epsilon_{Y}(\sigma(L))\right| \\
& +\left(\lambda_{i}\right)_{k 2}\left|\epsilon_{Y}\left(\sigma^{n-1}(X)\right) \cdot \epsilon_{X}(\sigma(L))+\epsilon_{Y}\left(\sigma^{n-1}(Y)\right) \cdot \epsilon_{Y}(\sigma(L))\right| \\
= & \left(D_{\sigma^{n}}\right)_{k \ell} .
\end{aligned}
$$

In contrast, if $\sigma$ is $z$-additive, then by Lemma A.6. $\epsilon_{L}(\sigma(X)) \cdot \epsilon_{L}(\sigma(Y)) \geq 0$. Then within each set of square brackets, the pair of products are actually of different signs, so that

$$
\begin{aligned}
\operatorname{row}_{k}\left(D_{\sigma^{n-1}}\right) \cdot \operatorname{col}_{\ell}\left(D_{\sigma}\right)= & \left(\lambda_{i}\right)_{k 1}\left|\epsilon_{X}\left(\sigma^{n-1}(X)\right) \cdot \epsilon_{X}(\sigma(L))-\epsilon_{X}\left(\sigma^{n-1}(Y)\right) \cdot \epsilon_{Y}(\sigma(L))\right| \\
& +\left(\lambda_{i}\right)_{k 2}\left|\epsilon_{Y}\left(\sigma^{n-1}(X)\right) \cdot \epsilon_{X}(\sigma(L))-\epsilon_{Y}\left(\sigma^{n-1}(Y)\right) \cdot \epsilon_{Y}(\sigma(L))\right| \\
\neq & \left(D_{\sigma^{n}}\right)_{k \ell} .
\end{aligned}
$$

Now let $\sigma$ be $x$-deficient. Then

$$
\begin{aligned}
\operatorname{row}_{k}\left(D_{\sigma^{n-1}}\right) \cdot \operatorname{col}_{\ell}\left(D_{\sigma}\right)= & {\left[\left(\lambda_{x}\right)_{k 1}\left|\epsilon_{X}\left(\sigma^{n-1}(Y)\right)\right|+\left(\lambda_{x}\right)_{k 2}\left|\epsilon_{Y}\left(\sigma^{n-1}(Y)\right)\right|\right] } \\
& \cdot\left(-\left|\epsilon_{X}(\sigma(L))\right|+\left|\epsilon_{Y}(\sigma(L))\right|\right) \\
& +\left[\left(\lambda_{x}\right)_{k 1}\left|\epsilon_{X}\left(\sigma^{n-1}(X Y)\right)\right|+\left(\lambda_{x}\right)_{k 2}\left|\epsilon_{Y}\left(\sigma^{n-1}(X Y)\right)\right|\right] \cdot\left|\epsilon_{X}(\sigma(L))\right| \\
= & \left(\lambda_{x}\right)_{k 1}\left[\left(\left|\epsilon_{X}\left(\sigma^{n-1}(X Y)\right)\right|-\left|\epsilon_{X}\left(\sigma^{n-1}(Y)\right)\right|\right) \cdot\left|\epsilon_{X}(\sigma(L))\right|\right. \\
& \left.+\left|\epsilon_{X}\left(\sigma^{n-1}(Y)\right) \cdot \epsilon_{Y}(\sigma(L))\right|\right]+\left(\lambda_{z}\right)_{k 2}\left[\left(\left|\epsilon_{Y}\left(\sigma^{n-1}(X Y)\right)\right|\right.\right. \\
& \left.\left.-\left|\epsilon_{Y}\left(\sigma^{n-1}(Y)\right)\right|\right) \cdot\left|\epsilon_{X}(\sigma(L))\right|+\left|\epsilon_{Y}\left(\sigma^{n-1}(Y)\right)\right| \cdot\left|\epsilon_{Y}(\sigma(L))\right|\right] .
\end{aligned}
$$

Now let $\sigma$ be $z$-additive. Then for $I=X, Y$,

$$
\left|\epsilon_{I}\left(\sigma^{n-1}(X Y)\right)\right|-\left|\epsilon_{I}\left(\sigma^{n-1}(Y)\right)\right|=\left|\epsilon_{I}\left(\sigma^{n-1}(X)\right)\right|,
$$

so that again in this case, we have

$$
\begin{aligned}
\operatorname{row}_{k}\left(D_{\sigma^{n-1}}\right) \cdot \operatorname{col}_{\ell}\left(D_{\sigma}\right) & =\left(\lambda_{z}\right)_{k 1}\left[\left|\epsilon_{X}\left(\sigma^{n-1}(X)\right) \cdot \epsilon_{X}(\sigma(L))\right|+\left|\epsilon_{X}\left(\sigma^{n-1}(Y)\right) \cdot \epsilon_{Y}(\sigma(L))\right|\right] \\
& +\left(\lambda_{z}\right)_{k 2}\left[\left|\epsilon_{Y}\left(\sigma^{n-1}(X)\right) \cdot \epsilon_{X}(\sigma(L))\right|+\left|\epsilon_{Y}\left(\sigma^{n-1}(Y)\right) \cdot \epsilon_{Y}(\sigma(L))\right|\right] .
\end{aligned}
$$

Noting that $x$-deficiency implies $\epsilon_{X}(\sigma(L)) \cdot \epsilon_{Y}(\sigma(L)) \geq 0$ by Corollary A.1, and $z$-additivity implies $\epsilon_{I}(\sigma(X)) \cdot \epsilon_{I}(\sigma(Y)) \geq 0$ by Lemma A.6 then within each set of square brackets, the products are of the same sign. Hence again we have

$$
\begin{aligned}
\operatorname{row}_{k}\left(D_{\sigma^{n-1}}\right) \cdot \operatorname{col}_{\ell}\left(D_{\sigma}\right)= & \left(\lambda_{i}\right)_{k 1}\left|\epsilon_{X}\left(\sigma^{n-1}(X)\right) \cdot \epsilon_{X}(\sigma(L))+\epsilon_{X}\left(\sigma^{n-1}(Y)\right) \cdot \epsilon_{Y}(\sigma(L))\right| \\
& +\left(\lambda_{i}\right)_{k 2}\left|\epsilon_{Y}\left(\sigma^{n-1}(X)\right) \cdot \epsilon_{X}(\sigma(L))+\epsilon_{Y}\left(\sigma^{n-1}(Y)\right) \cdot \epsilon_{Y}(\sigma(L))\right| \\
= & \left(D_{\sigma^{n}}\right)_{k \ell} .
\end{aligned}
$$

A similar calculation would hold in the case that $\sigma$ is $y$-additive and $x$-deficient. However, if $\sigma$ is $x$-deficient and $x$-additive, then for $I=X, Y$,

$$
\left|\epsilon_{I}\left(\sigma^{n-1}(X Y)\right)\right|-\left|\epsilon_{I}\left(\sigma^{n-1}(Y)\right)\right|=\left|\epsilon_{I}\left(\sigma^{n-1}(X)\right)\right|-2\left|\epsilon_{I}\left(\sigma^{n-1}(Y)\right)\right| .
$$

This extra term immediately implies that in this case

$$
\operatorname{row}_{k}\left(D_{\sigma^{n-1}}\right) \cdot \operatorname{col}_{\ell}\left(D_{\sigma}\right) \neq\left(D_{\sigma^{n}}\right)_{k \ell} \cdot
$$

The case where $\sigma$ is $y$-deficient is similar to the case for $x$-deficiency and is left to the reader. 


\section{REFERENCES}

[1] Bedford, E., and Smillie, J., Polynomial diffeomorphisms of $\mathbb{C}^{2}$. II: Stable manifolds and recurrence, Journ. AMS 4 no. 4 (1991), 657-679. MR1115786 (92m:32048)

[2] Bellon, M., and Viallet, C.-M., Algebraic Entropy, Commun. Math. Phys. 204 (1999), 425437. MR.1704282 (2000f:37040)

[3] Bonifant, A., and Fornaess, J., Growth of degree for iterates of rational maps in several variables., Indiana Univ. Math. J. 49 (2000), no. 2, 751-778. MR1793690 (2003g:32035)

[4] Brown, R., The polynomial degree of the special linear characters of a free group on two generators, preprint.

[5] Brown, R., Anosov mapping class actions on the $S U(2)$-representation variety of a punctured torus, Ergod. Th. \& Dynam. Sys. 18 (1998), 539-554. MR.1631712 (99j:58150)

[6] Cohen, M., Metzler, W, and Zimmerman, A., What does a basis for F $(a, b)$ look like?, Math. Ann. 257 (1981), 435-445. MR0639577 (82m:20028)

[7] Fornaess, J., and Sibony, N., Complex dynamics in higher dimension. II., Modern methods in complex analysis (Princeton, NJ, 1992), 135-182, Ann. of Math. Stud., 137, Princeton Univ. Press, Princeton, NJ, 1995. MR1369137 (97g:32033)

[8] Fornaess, J., and Wu, H., Classification of degree 2 polynomial automorphisms of $\mathbb{C}^{3}$, Publ. Math. 42 (1998), 195-210. MR1628170 (99e:14015)

[9] Fricke, R., Über die Theorie der automorphem Modulgrupper, Nachr. Akad. Wiss. Göttingen (1896), 91-101.

[10] Fricke, R., and Klein, F., Vorlesungen über die Theorie der automorphem Functionen, Band 1: Die gruppentheoretischen Grundlagen. Band II, Johnson, New York, 1965. MR0183872 $(32: 1348)$

[11] Fried, D., Word maps, isotopy, and entropy, Trans. AMS 296 no. 2 (1986), 851-859. MR0846609 (87k:58243)

[12] Friedland, S., and Milnor, J., Dynamical properties of plane polynomial automorphisms, Ergod. Th. \& Dynam. Sys. 9 (1989), 67-99. MR0991490 (90f:58163)

[13] Goldman, W., The modular group action on real SL(2)-characters of a one-holed torus, Geom. Topol. 7 (2003) 443-486. MR2026539 (2004k:57001)

[14] Goldman, W., and Neumann, W., Homological action of the modular group on some cubic moduli spaces, preprint.

[15] Gromov, M., On the entropy of holomorphic maps, Enseign. Math. 49 no. 3-4 (2003), 217-235. MR2026895 (2005h:37097)

[16] Guedj, V., and Sibony, N., Dynamics of polynomial automorphisms of $\mathbb{C}^{k}$, Ark. Mat. 40 (2002), 207-243. MR.1948064 (2004b:32029)

[17] Horadam, A., Basic properties of a certain generalized sequence of numbers, Fibonacci Quart. 3 (1965), 161-176. MR0186615 (32:4074)

[18] Horowitz, R., Characters of free groups represented in the two-dimensional special linear group, Comm. Pure Appl. Math. 25 (1972), 635-649. MR0314993 (47:3542)

[19] Maegawa, K., Quadratic polynomial automorphisms of dynamical degree golden ratio of $\mathbb{C}^{3}$, Ergod. Th. \& Dynam. Sys. 21 (2001), 823-832. MR.1836434 (2002e:32026)

[20] Maegawa, K., Classification of quadratic polynomial automorphisms of $\mathbb{C}^{3}$ from a dynamical point of view, Indiana Univ. Math. J. 50 (2001), 935-951. MR1864065 (2003b:37068)

[21] Morgan, J., and Shalen, P., Valuations, trees, and degenerations of hyperbolic structures I, Ann. Math. 120 (1984), 401-476. MR0769158 (86f:57011)

[22] Nielsen, J., Die Isomorphismen der allgemeinen unendlichen Gruppe mit zwei Erzeugenden Math. Ann. 71 (1918), 385-397. MR.1511907

[23] Smillie, J., The entropy of polynomial diffeomorphisms of $\mathbb{C}^{2}$, Ergod. Th. \& Dynam. Sys. 10 (1990), 823-827. MR.1091429 (92b:58131)

[24] Yomdin, Y., Volume growth and entropy, Israel J. Math. 57 no. 3 (1987), 285-300. MR0889979 (90g:58008)

Department of Mathematics, The Johns Hopkins University, 3400 North Charles Street, Baltimore, Maryland 21218-2686

E-mail address: brown@math.jhu.edu 